\title{
The sun as a significant agent provoking earthquakes
}

\author{
G. Anagnostopoulos ${ }^{1, a}$, I. Spyroglou ${ }^{1,2}$, A. Rigas ${ }^{1}$, P. Preka-Papadema ${ }^{3}$, \\ H. Mavromichalaki ${ }^{3}$, and I. Kiosses ${ }^{1}$ \\ ${ }^{1}$ Demokritos University of Thrace Telecommunications \& Space Science Sector, Xanthi, \\ Greece \\ ${ }^{2}$ Central European Institute of Tecnology (CETEC), Masaryk University, Brno 62500, \\ Czech Republic \\ ${ }^{3}$ National and Kapodistrian University of Athens Faculty of Physics, Athens, Greece
}

Received 7 October 2020 / Accepted 7 October 2020

Published online 19 January 2021

\begin{abstract}
In this paper we provide significant evidence that the sun is a principal agent provoking seismic activity. In particular the aim of the studies presented is to examine the possible relation of the coronal hole $(\mathrm{CH})$ driven high speed solar wind streams (HSSs) with seismicity We performed several statistical studies of solar space and seismological data between 1980 and 2017 as well as a study for a long time interval from the year 1900 until the year 2017. (A1) Concerning the period 1980-2017 among other results we found that the earthquakes (EQs) with $M \geq 83$ between 2010-2017 (including the catastrophic earthquakes of Japan 2011 (M91) Sumatra 2012 (M86) and Chile 2015 (M83)) occurred during times of large coronal holes as seen by the Solar Dynamics Observatory (SDO) satellite and were related with CH-driven HSSs observed by the ACE spacecraft several weeks or a few months before the EQ occurrences. (A2) Further research on the hypothesis of the possible HSS-EQ relationship revealed a surprising novel finding: a power spectrum analysis suggests that during the decay phase of the SCC22 and SC23 and at the maximum of SC23 the values of the global seismic $(M \geq 6)$ energy output shows a periodic variation of $\sim 27$ days, which is the mean rotational period of the Sun. (A3) Moderate (not strong) storms in general precede the great EQs. (B) The study of the data for the time interval 1900-2017 revealed that: (1) all of the giant $(M \geq 85)$ EQs occurred during the decay minimum and the rising phase of the solar cycle or in the maximum phase but at times of a strong reduction of the monthly averaged sunspot number: Chile M95 1960 EQ - Alaska M92 1964 EQ - Sumatra M91 2004 EQ (decay phase) Japan M91 2011 EQ (rising phase of the "strange" SC24) (2) the global energy release of all EQs with magnitudes $M \geq 55$ show the highest values during the decay phase of the solar cycle and in particular three years after the solar maximum and (3) a very significant negative correlation $\left(r_{S}=-042 p<10^{-4}\right)$ was found between the SSN and the number of earthquakes with $M \geq 7$ during the period 1930-2010 during times of moderate and high amplitude solar cycles. (C) Another result of our study is that the comparison of the yearly
\end{abstract}


numbers of great $(M \geq 7)$ EQs with the SSN fails to provide correct statistical results whereas this is possible for the global seismic energy or the giant EQs. (D) Finally we infer that the case and statistical studies presented in this paper strongly suggest a close relation between $\mathrm{CH}$-associated HSSs and seismic activity. We present some observational evidence that most probably Alfvèn waves mediate the interaction of $\mathrm{CH}$-driven HSSs with seismicity.

\section{Introduction}

The Sun is the principal energy source in our solar system and, therefore, the agent of many physical processes taking place in Earth's magnetosphere $[11,100,131]$, ionosphere $[2,94]$, atmosphere [43,51,91], lithosphere $[5,82,110]$ and biosphere $[15,84]$. Moreover, nowadays our knowledge about the Sun-Earth electromagnetic relationship has continuously increased and allows a new insight into important physical and biological phenomena.

On Earth, earthquakes (EQs) are known to be the most catastrophic physical phenomenon. Earthquakes cause dramatic effects, such as thousands of deaths and a very high number of injured and homeless people every year, as well as social effects including destroyed cities and societal disruption. Besides, civil construction regulations nowadays scientific earthquake prediction research seems to be a hopeful social-scientific tool in reducing dramatic EQ effects. Significant progress towards a deeper understanding of the seismic phenomenon has been achieved by searching various electromagnetic (EM) phenomena preceding EQs ([5]; Athanasiou et al., 2012, $[8,33,35,95])$, which have been considered to be EQ precursors.

Furthermore EQs have been hypothesized to be related with Solar and space weather activity. Several studies have provided significant evidence that seismicity depends on solar cycle phase, solar wind and geomagnetic activity $[12,41,59,82,110$, $117,128,138]$. Furthermore, several studies have provided significant evidence that most of the EQs occur outside the maximum phase of the solar cycles, in particular during the decay phase of the solar cycle $[110,113,115]$.

Anagnostopoulos and Papandreou [5] examined space and seismological data in the decay phase of solar cycle \#23, including the time of occurrence of the M9.1 (M9.3) Sumatra catastrophic EQ/tsunami (December 26, 2004) and they found that a sequence of high speed streams (HSSs) of solar wind and subsequent geomagnetic disturbances systematically preceded a sequence of strong EQs throughout the month before the giant EQ of December 26, 2004. Figure 1b, which has been adapted from the study of Anagnostopoulos and Papandreou ([5]; their Fig. 5), compares the $M \geq$ 6.2 earthquakes with the difference $\Delta K p$ of the successive values of the 3 -h index $\overline{K p}$ of geomagnetic activity. An evaluation of the time difference $t=T_{E Q}-T_{M}$, between the EQ occurrence time $T_{E Q}$ and the time $T_{M}$ of the last peak with $\Delta K p \geq 2$ (but not $\geq 3$ ) before the Sumatra EQ suggested that $t$ varies in a small range of time, with $t=0-42 \mathrm{~h}$. The average value of the time difference $\mathrm{t}=T_{E Q}--T_{M}$ was estimated to be $t=34.9 \mathrm{~h} \approx 35 \mathrm{~h}$, that is about 1.5 days after the increase of the geomagnetic activity, while the times of the geomagnetic index increases $\Delta K p$ and the EQ occurrences were found to be strongly related $(r=0.9)$ at the significant level $p=0.02$ ([5] Fig. 6). (A few $\Delta K p$ peaks were not followed by an EQ with magnitude $M \geq 6.2 \mathrm{EQ}$, but each one of those EQs was preceded by a peak of $\Delta K p$ before the Sumatra earthquake). U-Yen [127] meanwhile has noted that the HSSs at those times were related with a great coronal hole $(\mathrm{CH})$, observed by SOHO in EUV band (Fig. 1a). However, despite the existing evidence, the possibility of an association of

a e-mail: ganagno@ee.duth.gr 
(a)

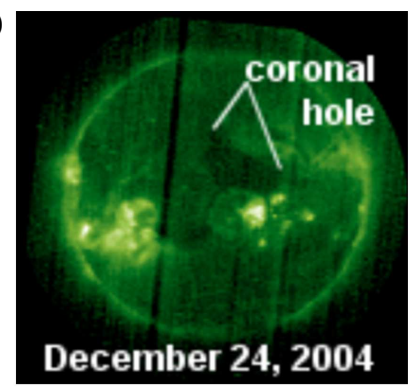

(b)

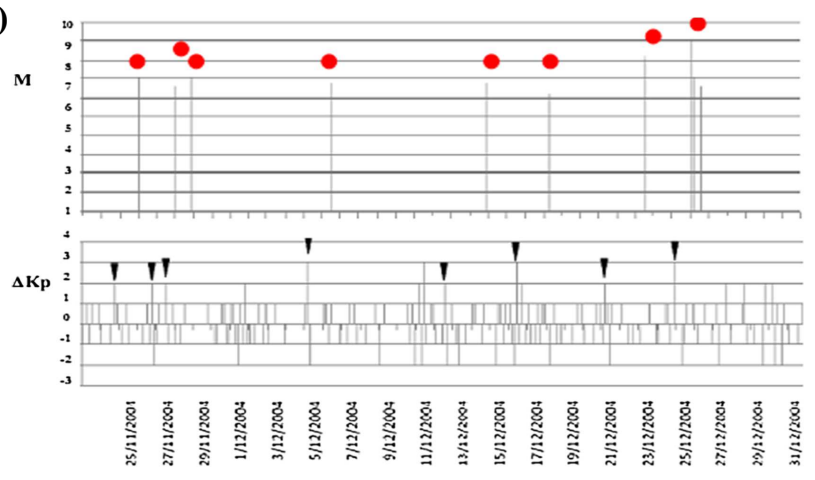

Fig. 1. (a) Solar, geomagnetic and seismological data before the catastrophic M9.1 SumatraAndaman giant earthquake (EQ) occurred on December 26, 2004. (a) A large coronal hole $(\mathrm{CH})$ observed by $\mathrm{SOHO}$ about two days before the giant earthquake (adapted from U-Yen (2015)); CH is a source of high speed solar wind streams (HSSs). (b) The magnitude of the great $(M>6.2)$ earthquakes along with the difference $\Delta K p$ of successive values of the geomagnetic 3-hour $\Delta K p$ index. The time of the earthquake occurrence is very well related $(r=0.9 ; p=0.02)$ with the time of the last maximum $\Delta K p$ before the corresponding $\mathrm{EQ}$, with an average time delay of $\sim 1.5$ days. The sequences of the HSSs led to SumatraAndaman giant EQ (Anagnostopoulos and Papandreou, 2012).

large EQs with solar activity has been disputed by Love and Thomas [69], within a framework of certain hypotheses.

Given that in our case study of the M9.1 (M9.3) Sumatra 2004 catastrophic EQ we found that the time-sifted sequences of HSSs and great EQs before the giant one of December 26 [5] which occurred during the decay phase of Solar cycle 23, at a time of a large coronal hole, we wanted to examine the hypothesis that such solarspace conditions show a systematic relationship with the giant EQs and with global seismicity.

Until now, the possible solar-terrestrial triggering of Earthquakes has been basically examined on the basis of a comparison between seismological data and the Sunspot Number (SSN). Although the SSN is a useful and the most known index of the solar activity, it is not the sole solar index. Moreover, the observed value of SSN does not reveal the whole status of the solar activity, in particular when long time (one year) data averages are used (this fact will be explained later in the paper).

On the other hand, the HSSs emanating from the solar corona is a significant agent, which is known to affect the Earth's environment (Sects. 2 and 3). Therefore, in this paper we investigate, beside the 11-year cycle of the SSN, the possible relationship of the CH-driven HSSs with seismic activity, and, in particular, the possible effect of the $\sim 27$-day mean solar rotation period on seismological data. For this reason, after a description of the instruments used in this study (Sect. 4), in Section 5 
we present the results from various statistical studies of giant $(\geq 7.8,8.3,8.5)$ EQs and of the global energy release from EQs with $M \geq 5.5$ (Sect. 5). Statistical results are shown from a period as long as 117 years (1900-2017), but detailed work focus on the study the four last solar cycles (1980-2017).

A variety of studies presented in this paper provide good evidence that $\mathrm{CH}$-driven HSSs (most often during the decay phase of the solar cycle) make a significant contribution in provoking giant earthquakes and enhance global seismicity. In Section 6 we discuss various issues relating to the new concept of the $\mathrm{CH}$-driven HSSs as a significant external agent of Earth's seismicity.

\section{Observational and theoretical space physics framework}

Fast solar wind streams are often observed not only in the near-Earth space, but also in the deep interplanetary space. These solar wind streams and their associated structures have been the subject of great interest as they, for example, affect space weather (e.g. see, $[10,18,56,65,77])$, influence geomagnetic activity (e.g., $[40,60,65,75$, $101,124]$ and references therein) and ionospheric variations from high to equatorial latitudes [2].

The most dramatic effect of the Sun in the interplanetary space is the Coronal Mass Ejections (CME). CMEs are large expulsions of plasma and magnetic field from the Sun's corona, which are released into the solar wind transferring billions of tons of coronal material. CMEs most often originate from active regions on the Sun's surface, such as groupings of sunspots, and they often follow solar flares. CMEs most often occur during solar maxima $[11,100]$. On the other hand, coronal holes (CH) are the least active regions of the Sun and they are seen as the darkest patches on the solar surface, as measured in ultraviolet (UV) and X-ray radiation. Coronal holes are regions of low density (low temperature) plasma on the Sun. They have magnetic fields opening freely into the heliosphere and they are associated with rapidly expanding open magnetic fields and the acceleration of high-speed solar wind. A more theoretical usage equates "coronal holes" with all open-field footprints of time-steady solar wind flows [42].

Despite the fact that dramatic solar flares or large CMEs-associated geomagnetic storms are known to drive spectacular effects in the Earth's near-space environment ([87] and references therein), the importance of the $\mathrm{CH}$-driven high speed streams (HSSs) for geomagnetic activity has also become increasingly accepted across the space community over the past few years. Various aspects of the physics of solar wind streams and their influence on the Earth's environment can be found in the AGU collection of papers in Geophysical Monograph \#167 edited by Tsurutani et al. [123] and in several specialized books and papers $[1,2,25,26,38,62,86,100-103,121-$ $123,131]$, as well as in references therein).

"Slow" solar wind originates from low latitude regions of the solar disk and is observed in the Earth's orbit at speeds of $\sim 300-400 \mathrm{~km} / \mathrm{s}$. "Fast" solar wind emanates from coronal holes on the Sun and is observed at Earth with velocities as high as $800 \mathrm{~km} / \mathrm{s}$; coronal holes are most prominent in polar regions during low solar activity.

The flow of plasma from the solar corona is non-uniform in both time and space. Observations from Ulysses, the first spacecraft that investigated the three dimensional heliosphere [89], confirmed a latitudinal dependence of the solar-wind speed. The fast solar wind catches up with upstream slow solar wind and a compressive region is formed at the interface of the two streams. These structures reappear with the $\sim 27$ days' rotation period of the Sun. When these coronal hole-associated streams are long lasting, they lead to the formation of corotating interaction regions (CIRs) in the interplanetary space and when the CIRs are well developed, they are bound by 


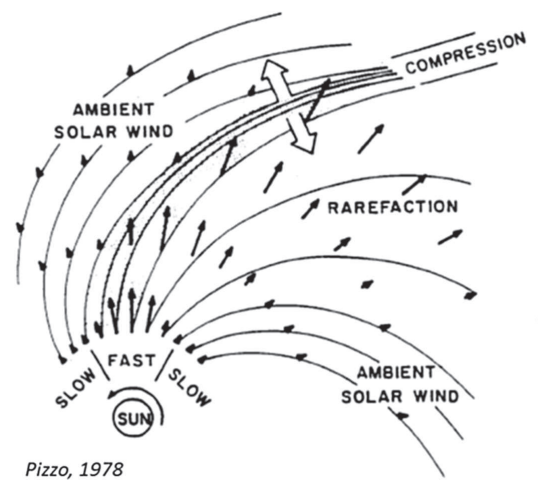

(a)

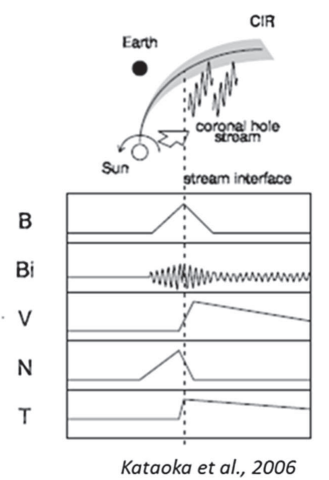

(b)

Fig. 2. A schematic presentation showing the formation of HHSs and co-rotating interaction regions (CIRs) and the variation of the most important solar wind parameters. (a) The flow of plasma from the solar corona is non-uniform in both time and space. "Fast" solar wind emanates from coronal holes on the Sun and catches up with upstream slow wind and forms a compressive region. When these coronal hole-associated streams are long lasting, they lead to formation of CIRs. (b) Typical variations of plasma parameters of a CIR: an increase in the solar wind plasma density $(N)$ and the interplanetary magnetic field $(B)$ due to the "pile-up" of material at the leading edge of the fast wind that is followed by a long lasting increased solar wind speed and the presence of large amplitude long low frequency (Alfvén) waves. HSSs/CIRs most often reappear within the $\sim 27$ - day rotation period of the Sun.

fast forward (FS) and fast reverse (RS) shocks. A schematic showing the formation of CIRs can be seen in Figure 2a (right side; adapted from [90]).

Typical variations of plasma parameters of a CIR are indicated in Figure 2b: we can see an increase in the solar wind plasma density $(\mathrm{N})$ and the interplanetary magnetic field (B) due to the "pile-up" of material at the leading edge of the fast wind, which is characterized by the presence of large amplitude long low frequency Alfvén waves; the compression of the interplanetary magnetic field is followed by the long lasting HSS.

HSSs dominate in the declining phase and close to the solar minimum of the solar cycle, when polar CHs extend towards the ecliptic plane, and can drive more important physical processes over longer periods than the more transient CME-driven geomagnetic storm can $[18,57,123]$. Previous observations of $\sim 27$ days periodicities in interplanetary plasma and field features have suggested that slowly evolving conditions of the coronal field topology produce an almost rigidly corotating solar wind structure during the declining phases of solar cycles [73].

The studies by Simpson [110], Anagnostopoulos and Papandreou [5], Kovalyov and Kovalyov [64], U-Yen [127], Gulyeva [45] among many other studies provide significant evidence that CH-driven HSSs may be an agent provoking earthquake occurrence. Therefore, we wanted to further check whether repetitive $\mathrm{CH}$-driven HSSs occurring in the Earth's environment provide sufficient physical conditions (i.e. transferring of electromagnetic energy) for enhanced seismicity. For this reason, the scenario of the possible relationship between $\mathrm{CH}$-driven HSSs and global seismic energy is examined in the present paper extensively by performing various statistical studies of space and seismological data, in comparison with solar sunspot numbers and EUV solar images (Sect. 5). We also reexamine some previously published results and we explain why some authors considered that the solar-terrestrial triggering is insignificant (Sect. 6). 


\section{Magnetosphere-ionosphere-lithosphere electromagnetic coupling}

The ionosphere is a plasma region near Earth which is highly sensitive to space plasma variations and to geomagnetic storms. In the ionosphere, the solar wind generates electrical currents. On the Earth's surface these currents cause magnetic field fluctuations. These fluctuations penetrating the Earth's interior induce the electrical currents $J$ and in the presence of the Earth's magnetic field $B$ generate an electromagnetic force known as the Lorentz force $F=J \times B$ [128]. However, despite significant observational evidence provided by many researchers that there is a relation between geomagnetic and seismic activity the subject still remains controversial in scientific community and there is still not a well-determined and generally accepted theoretical framework to explain this interaction.

Sobolev et al. [112] and Zakrzhevskaya and Sobolev [138] have argued that if the crust in the area of a forthcoming EQ is in a metastable state, it becomes sensitive to trigger-like effects. They speculated that the electrical energy supplied to the Earth during a magnetic storm is converted into mechanical energy via piezoelectric, electrokinetic or other mechanoelectric effects and increases mechanical stresses. They noted that a comparison of the energy supplied by magnetic storms and that released by EQs suggests that a magnetic storm can only act as a triggering mechanism, not as the main cause of the seismic activity. On the contrary, Duma and Ruzhin [29] argued that the magnetic moment, generated by the induced ionospheric Sq current system, interacts with the horizontal intensity $\mathrm{H}$ of the main Earth's magnetic field and the torque resulting from this process acts on the current sheet (on the lithosphere) and can produce EQs with magnitudes up to $M=6$.

Some authors suggested other extraterrestrial processes as triggering mechanisms of seismic activity: solar and lunar tides [53], solar proton fluxes [129], and earthward movement of the magnetopause, the boundary between the Earth's magnetic field and the solar wind [74]. Kormiltsev et al. [63] hypothesized that magnetic storms induce electro-osmotic fluid flows which cause EQ triggering due to the influence of anomalous porous pressure. Sytinskii [116] argued that the EQ triggering mechanism is the solar- induced change in atmospheric circulation expressed in the large scale reorganization of baric fields, while Prikryl et al. [93] provided evidence that atmospheric gravity waves are generated by auroral electrojets caused by high speed solar wind MHD waves.

The Sun-interplanetary space - magnetosphere - ionosphere - atmosphere lithosphere chain is a complicated, open, dynamic, nonlinear system including complex processes, with high unpredictability. In this context, the seismic phenomena of the Earth should be considered as a part of the whole Sun-Earth system. The relation between seismicity and the Sun-Earth chain processes has been considered as ambiguous, and for this reason the contribution of various solar impact processes on the Earth's lithosphere and their association with the seismic energy release needs more work.

In the present paper we present some new observational results, which not only confirm a relationship between Sun, magnetosphere and lithosphere, but provide some special information, which probably indicates a theoretical research direction to understand some basic parts in the physical chain of the Sun-lithosphere electromagnetic coupling. As we will see in the next sections, the HSSs/CIRs seem to play an important role in triggering EQs. 


\section{Data}

In order to test the hypothesis that seismicity is related to $\mathrm{CH}$-driven HHSs/CIRs during small to moderate SSNs and, in particular, during the decay phase of the solar cycle, we combined solar, interplanetary, geomagnetic, and seismological data.

Observations obtained by the SDO and the ACE satellites were used in order to analyze observations from the solar corona activity and the near Earth's space, respectively.

The Solar Dynamics Observatory (SDO) was designed to understand the causes of the Sun's variability and its impacts on Earth and it was launched on February 11, 2010 (https://sdo.gsf c.nasa.gov/). SDO is the first satellite launched for NASA's Living With a Star (LWS) Program. The goal of this mission is to advance the predictive capability of solar influence on Earth's humanity and humanity's technological systems. SDO moves at a circular geosynchronous orbit inclined at $28^{\circ}$ about the longitude of a dedicated ground station in New Mexico [88].

The SDO mission includes three scientific investigations: the Atmospheric Imaging Assembly (AIA), Extreme Ultraviolet Variability Experiment (EVE), and Helioseismic and Magnetic Imager (HMI) [88]. The AIA telescope takes images from the Sun in 10 wavelengths every 10 seconds. For the needs of the present study, the solar corona activity was examined by using AIA images obtained in the passband of the electromagnetic spectrum at $193 \AA\left(10^{-10} \mathrm{~m}\right)$ every one hour (https : //helioviewer.org).

The Advanced Composition Explorer (ACE) is a spacecraft, which has been circulating around the $L_{1}$ Lagrangian point, which is the point of Earth-Sun gravitational equilibrium at a distance of some 1.5 million $\mathrm{km}$ from the latter, or $\sim 220$ $R_{\mathrm{E}}$, (where $R_{\mathrm{E}}$ is the length of Earth's radius). The prime objective of ACE is to measure and compare the composition of several samples of matter, including the solar corona, the solar wind, and other interplanetary particle populations. The ACE scientific research programme includes eight instruments that measure plasma and energetic particle composition and one to measure the interplanetary magnetic field [109].

When reporting space weather ACE, provides an advance warning (about one hour) of geomagnetic storms that can overload power grids, disrupt communications, and present a hazard to astronauts. ACE was launched on August 25, 1997 from the Kennedy Space Center in Florida and has been continuously providing in situ observations until the present. Real-time observations with 1 second resolution are provided continuously to Space Environmental Center (SEC) of the National Oceanographic and Atmospheric Association (NOAA). For the present study we used data from the ACE Level 3 Summary Plots (http://www.srl.caltech.edu/ACE/ASC/ DATA/level3/summaries.html).

ACE data were used from the ACE/MAG instrument observing magnetic field (http://www.ssg.sr.unh.edu/mag/ACE.html) and from Solar Wind Electron, Proton and Alpha Monitor (SWEPAM) gathering bulk plasma data (http://www.srl. caltech.edu/ACE). Although magnetic field and solar wind data provide sufficient information for identifying various space structures, such as the CMEs and CIRs, the analysis of energetic particle data allow a better insight into these structures. For this reason, data from the Electron, Proton, and Alpha Monitor (EPAM) particle instrument (http://sd-www.jhuapl.edu/ACE/EPAM) were also used in order to fully investigate the space weather before great EQs. More information on instruments on ACE can be found in the ACE site (http://www.srl.caltech.edu/ACE/). The sunspot number was obtained by WDC-SILSO, Royal Observatory of Belgium, Brussels (http://www.sidc.be/silso/datafiles). 


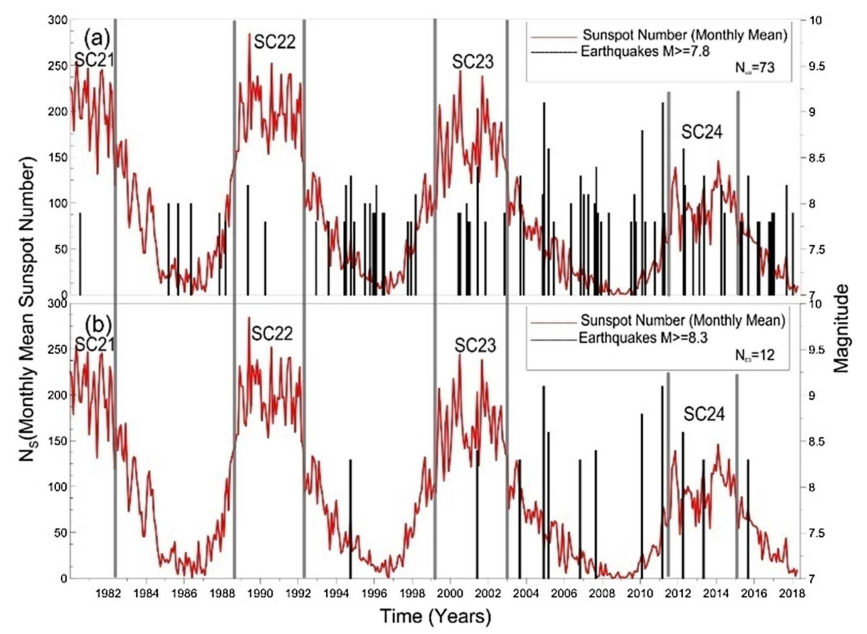

Fig. 3. The monthly mean of the sunspot number (SSN) along with the EQs with magnitude $M \geq 7.8$ (panel a) and $M \geq 8.3$ (panel b) occurred between 1980 and 2017. The grey normal lines have been drawn to indicate the maximum phase of each solar cycle (see Sect. 5.1). In Figure 3a, we see that the $M \geq 7.8$ EQs are assembled into several groups. Most of the EQs are seen during moderate and low values in both panels, and in particular during the decay phase and close to solar minimum (see Fig. 5a). During the decay phase of the solar cycle, when the frequency of EQs was recorded high, the frequency of HSSs emanating from the solar corona also got the highest values.

The geomagnetic three-hour index $\mathrm{Kp}$, which is presented in our data analysis as well as other geomagnetic indexes we advised, were obtained from the World Data Center for Geomagnetism, Kyoto, Japan (http://wdc.kugi.kyoto-u. ac.jp/kp/index.html). Data concerning EQ information were used from the sites U.S. Geophysical (http://earthquake.usgs.gov/earthquakes/search) survey as well as that of the European-Mediterranean Seismological Society (https://www . emsc-csem.org).

\section{Observations and results}

\subsection{Solar and intense earthquake $(M \geq 7.8,8.3)$ activity during solar cycles 21-24}

In Figure 3 we compare the variation of the sunspot number (SSN) with big EQ occurrences during the four last solar cycles: SC21, SC22, SC23, and SC24. From Figure 3 we see that SC21 - SC24 show a decreasing trend in the maximum SSN of the successive solar cycles. In addition, during the minimum transit SC23 to SC24, the solar observations revealed the longest spotless periods since the beginning of daily solar observations in 1849 . It is pointed out that during the minimum transit from solar cycle 23 to 24 , there was a total of 817 days with no sunspots and that the solar cycle \#23 was the longest solar cycle in 180 years (http://users.telenet. $\mathrm{be} / \mathrm{j} \cdot \mathrm{janssens/Spotless/Spotless.html \# Main).}$

The upper panel of Figure 3 (panel a) displays the monthly mean of the sunspot number $N_{s}$ along with the EQs with magnitude $M \geq 7.8$ between 1980 and 2018 (January); the length of the vertical lines within the panel represents the magnitude of the corresponding earthquake. The left vertical axis indicates the sunspot number 
Table 1. The number of earthquakes with magnitudes $M \geq 7.8$ occurred in the period 1980-2018 (January) during the maximum phases of solar cycles 21-24 and the transits from one cycle to the next. Most of the EQs (77\%) are seen outside the periods of solar maxima.

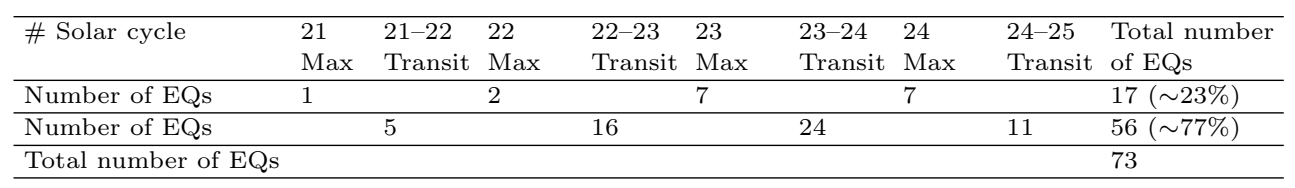

Table 2. The number of EQs considered in Table 1, which occurred during the transits from one cycle to the next; the sample is here examined by comparing the number of EQs happening in the rising versus the decay phase of each cycle. We see that most EQs $(77 \%)$ occurred during the decay phase.

\begin{tabular}{lll}
\hline \# Solar cycle & Rising phase & Decay phase \\
\hline 21 & - & 3 \\
22 & 2 & 13 \\
23 & 3 & 16 \\
24 & 8 & $(11)$ \\
\hline (1980-2018) Jan & $\mathbf{1 3}$ & $\mathbf{4 3}$ \\
& $\mathbf{( 2 3 \% )}$ & $\mathbf{( 7 7 \% )}$ \\
\hline
\end{tabular}

$N_{s}$ while the one on the right the EQ magnitude $M$. In Figure 3a, we see that the strong $(M \geq 7.8)$ EQs are assembled into several groups.

In Tables 1 and 2 we examine the distribution of EQs presented in Figure $3 \mathrm{a}$ in reference to the solar cycle phase. Table 1 shows the number of EQs occuring in the maximum phase of solar cycles, in comparison to the number of EQs which occurred during the transits from one cycle to the next. In particular, data are shown from the maximum phase of SC21 up to the transit of SC24 to SC25 (notice that this phase is still in progress, so the corresponding number of EQs during this last period may not be a final number). From Table 1 it is clear that a total of $17 M \geq 7.8$ earthquakes occurred during the maximum phases, whereas a number of EQs as high as 56 happened during the periods of the transits from one cycle to the next. These numbers, 17 versus 56, correspond to a percentage of $23 \%$ and $77 \%$ of the total number (73) of EQs examined in Figure 3a.

In Table 2 we elaborate the distribution of the above subtotal of 56 big EQs in reference to the rising and decay phase of the solar cycles 21 to 24 . We see that a total of 13 EQs occurred in the rising phase of SC21-SC24, whereas a much greater number, a number as high 43 EQs, occurred during the decay phase of these solar cycles.

From Tables 1 and 2 we infer the following for the EQs with magnitude $M \geq 7.8$ occurred during the period 1980-2018 (January): (i) there is a tendency of the big EQs to occur during the transit from one cycle to the next compared to the maximum phase and (ii) when examining the EQs which occurred during the transit from one cycle to the next, we see a much greater percentage (77\%) of EQs occuring in the decay phase compared to that of EQs occuring in the rising phase of solar cycles. Two more comments can be made concerning the distribution of big EQs between the years 1980-2018 (Jan.). Firstly, as we can see from both Tables 1 and 2, the number of EQs, in each phase, increases in general with time from the maximum of SC21 to the maximum of SC24. Secondly, a more detailed data analysis that we made suggests that three out of seven big EQs seen during the maximum of SC23 (Fig. 3a) occurred in November 2000, when the SSN decreased from a value of 244 


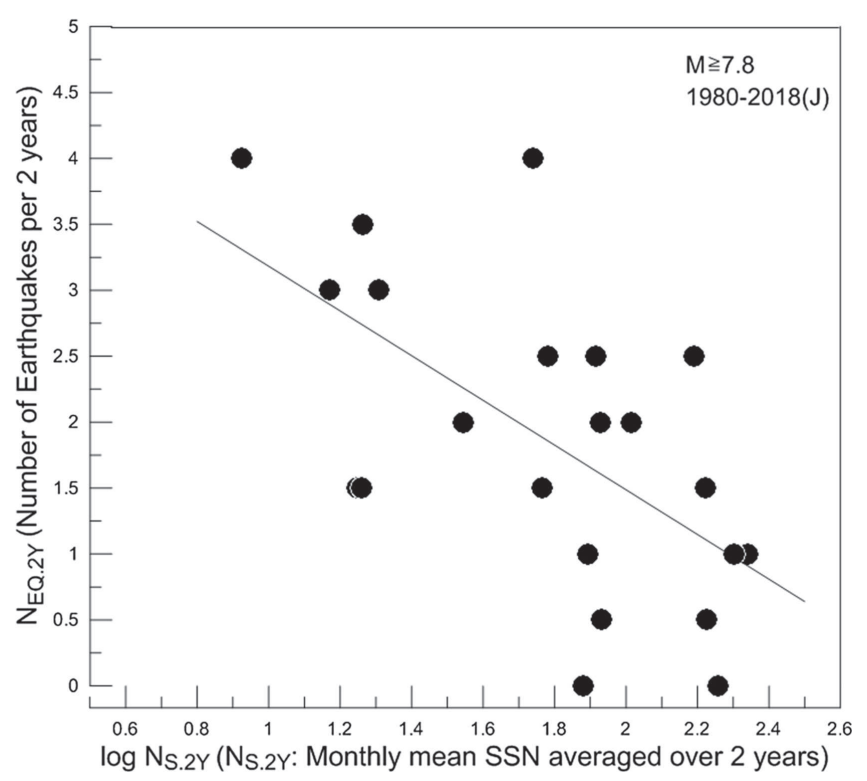

Fig. 4. The number of EQs $N_{E Q, 2}$ with magnitudes $M \geq 7.8$ averaged over two years versus the logarithm of the monthly mean SSN averaged over the corresponding two year-interval, between 1980-2018 (Jan.). We clearly see a negative trend of association between the two quantities $\left(r_{s}=-0.55, P=0.016\right)$. These data suggest that during the whole time interval of four recent solar cycles (SC21-SC24) there is a trend towards a higher frequency of a big $(M \geq 7.8)$ EQ when the SSN decreases.

in July 2000 to a local monthly minimum value as low as 158 in November 2000; the three big EQs occurred within a period of only two days (2000.11.16, M8; 2000.11.16, M7.8; 2000.11.17, M7.8) in the New Britain/New Ireland region of Papua New Guinea [85]. The above results suggest that there is a tendency of big EQs to occur during times of moderate to low SSNs, and, in particular, during the decay phase of the solar cycle; more analysis is needed in order to examine the possible relation of EQs occurring during the maximum phase at times of large decreases in the SSN during the maximum phase (as in the case of EQs in November 2000).

In Figure 4 we display the number of EQs $N_{E Q, 2}$ with magnitudes $M \geq 7.8$ averaged over two years versus the logarithm of the monthly mean SSN averaged over the corresponding two year-interval, between 1980-2018 (Jan.). From Figure 4 we clearly see a negative trend of association between $N_{E Q, 2 Y}$ and $\log N_{s, 2 Y}$, with a moderate Spearman correlation coefficient $r_{s}=-0.55$, but significant at a level $P=$ 0.016. The data shown in Figure 4 suggest that during the whole time interval of the four recent solar cycles (SC21 - SC24) there is a clear trend towards higher EQ frequency when the sunspot number $N_{S, 2 Y}$ decreases. This finding is in agreement with the results of Rekapalli [97], who used a Singular Spectral Analysis and correlation methods and found that there was a linear increase in the yearly earthquake number from 1975 to 2005.

In order to further examine whether the observed large number of earthquakes in the transit phase is statistically significant, we conducted a hypothesis test, by shuffling randomly 1000 times the earthquake dates in accordance with a uniform random distribution in the range of the years 1980-2018. For every sample we calculate the number of earthquakes in the transit phase and as a result we can get the distribution of this metric (approximate Gaussian). Figure 5a shows that the real number of earthquakes is significant at a 95\% confidence level (vertical black lines), because the real number of earthquakes during the transit phases (56) is larger than 
the mean value (49.9) plus 1.96 times the standard deviation (3.57). This result suggests that the hypothesis that there is a tendency of the big EQs to occur during the transit from one cycle to the next compared to the maximum phase cannot be rejected $(p<0.05)$.

Moreover, to further ensure the statistical significance of the reported correlation shown in Figure 4, we performed an additional statistical test. We bootstrap the data points 1000 times and fit the linear trends. As a result, we get the sampling distribution of the observed slope shown in Figure 5b. We can see that the estimated value of the slope $(-0.01929)$ is statistically significant at $95 \%$ confidence level (vertical black lines), as the mean value of the sampling distribution minus 1.96 times the standard deviation is equal to -0.0175 . This result suggests the hypothesis that there is a clear trend towards higher EQ frequency $N_{E Q, 2 Y}$ when the sunspot number $N_{S, 2 Y}$ decreases.

Here, we would like to point out a limitation to a simple negative correlation between the SSN and the number of big earthquakes described above. An elaboration of the data in Figure 4 suggests that, during the minimum phase of SC22 (July 1996 Sept. 1997) and SC23 there are no big $(M \geq 7.8)$ EQs. This absence of big EQs at those times is related to the arrival of solar wind with the lowest values measured from 1975 until 2017 [119,131]. Luhmann et al. [73] suggested that the cause of the unusual low solar wind speed during the $23 / 24$ minimum is the underlying weak solar polar field. Therefore, we infer that the correlation of the SSN with earthquakes in general applies, with certain limitations, as for instance during periods of very low SSN. Such a behavior with low seismic activity during low SSN is known as the Maunder (1645-1720) and Dalton (1790-1820) minima (Sect. 6.2).

Figure $3 \mathrm{~b}$ has been constructed as Figure 3a, but for giant EQs with a magnitude as high as $M \geq 8.3$. The most important characteristic features in the frequency of twelve giant EQs which occurred in the whole time period examined (19802018/January) are the following: (a) There is no giant earthquake occuring in the first $\sim 14.75$ years, during intense solar activity (during SC21 and SC22), (b) a high occurrence frequency of giant EQs can be seen between 2003-2010, with 6 EQs out of the total of the $12 \mathrm{EQs}$ - spread over 6.5 years, during the minimum transit from SC23 to SC24. We infer that the occurrence times of the giant EQs in Fig. 3b are consistent with the trend of $M \geq 7.8$ EQs for higher frequency during the decay phase and close to the solar seen in Figures $3 \mathrm{a}$, 4a, and 5 . In addition we see a distinct gathering of giant EQs in the decay phase of SC23 and during SC24, which was a period with "peculiar" solar and interplanetary activity (see Sect. 5.2).

\subsection{Solar and seismic activity $(M \geq 5)$ during solar cycles 23 and 24}

In this section we concentrate on the SC23 and the SC23-SC24 transition, when special solar and seismic activity were recorded. As we showed in the previous section many giant earthquakes occurred during the deep solar minimum following the SC23 maximum phase.

The solar cycle 23 lasted 12.3 years, beginning in August 1996 and ending in December 2008, and it is one of the longest solar cycles from the beginning of daily solar observations in 1849. Furthermore, the SC23-SC24 minimum transit was one of the longest since 1849, with a spotless number as high as 817 days. As we have already mentioned in the paper by Anagnostopoulos and Papandreou [5], during the descending phase of SC23, a series of fast solar wind streams for about one month was followed by another series of large EQs, shifted in time by an average of 1.5 days, which finished with the M9.1 Sumatra-Andaman earthquake and the associated catastrophic tsunami of December 26, 2004. 

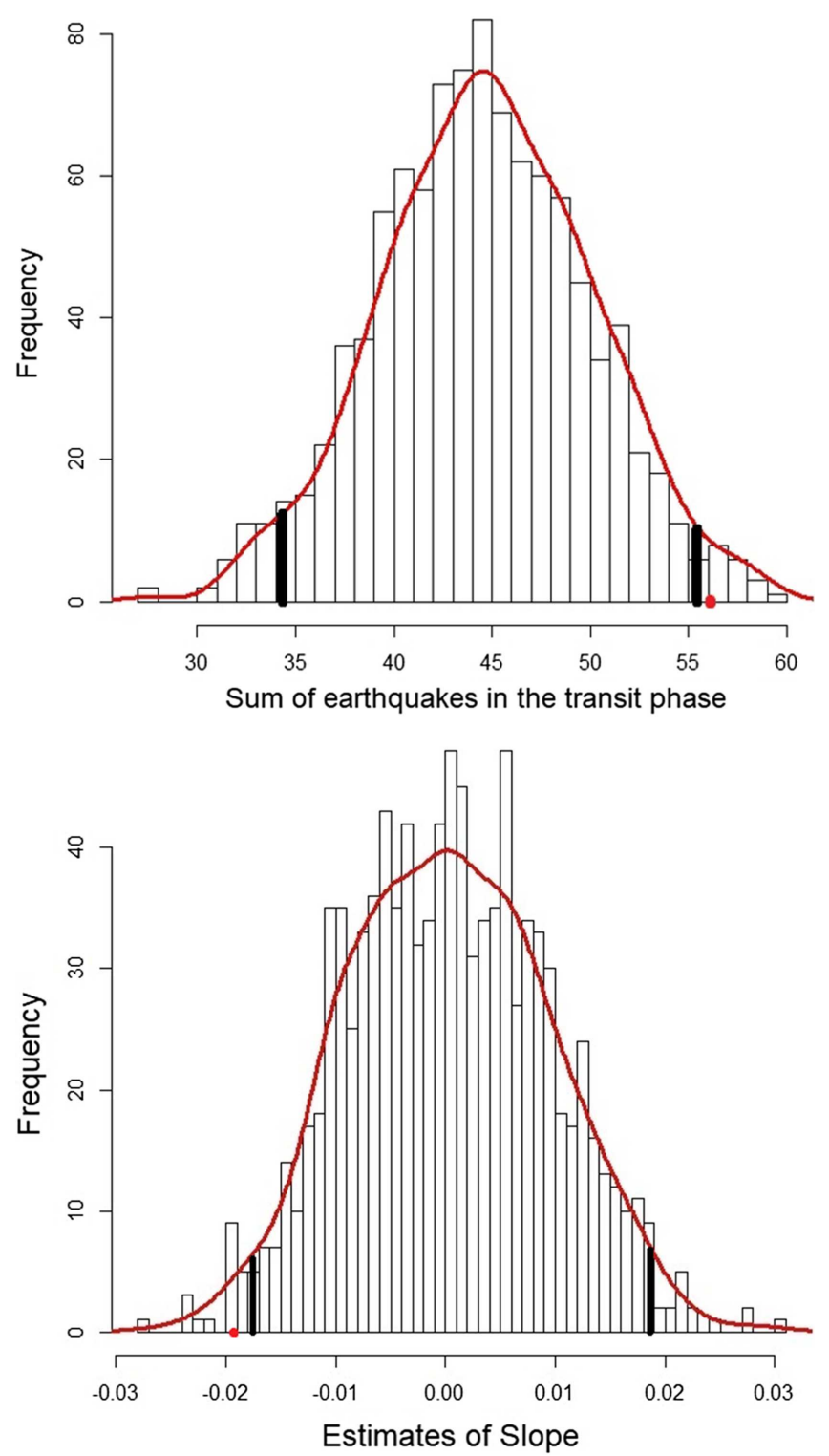

Fig. 5. (a) Histogram of sampling distribution of number of earthquakes in the transit phase obtained by shuffling randomly 1000 times the earthquake dates in accordance with a uniform distribution in the period of 1980-2018. The red curve indicates the Gaussian distribution that approximates nicely the histogram of the sampling distribution. The vertical black lines indicate the $95 \%$ confidence interval. Figure 5a suggests that the null hypothesis that more earthquakes in the time interval 1980-2018 (Jan.) occur in the transit from one cycle to the next rather than during the maximum phase cannot be rejected $(P<0.05)$. (b) Histogram of the sampling distribution of the observed slope of the data of Figure 5 obtained by bootstrapping the data points 1000 times and fitting the linear trends. The red curve indicates the Gaussian distribution that approximates quite well the histogram of the sampling distribution. This figure indicates that the number of EQs $N_{E Q, 2}$ with magnitudes $M \geq 7.8$ averaged over two years and the logarithm of the monthly mean SSN averaged over the corresponding two year-interval $\log N_{s, 2 Y}$ between 1980-2018 (Jan.; Fig. 4) shows a negative correlation, which is significant at a level $P<0.05$. 

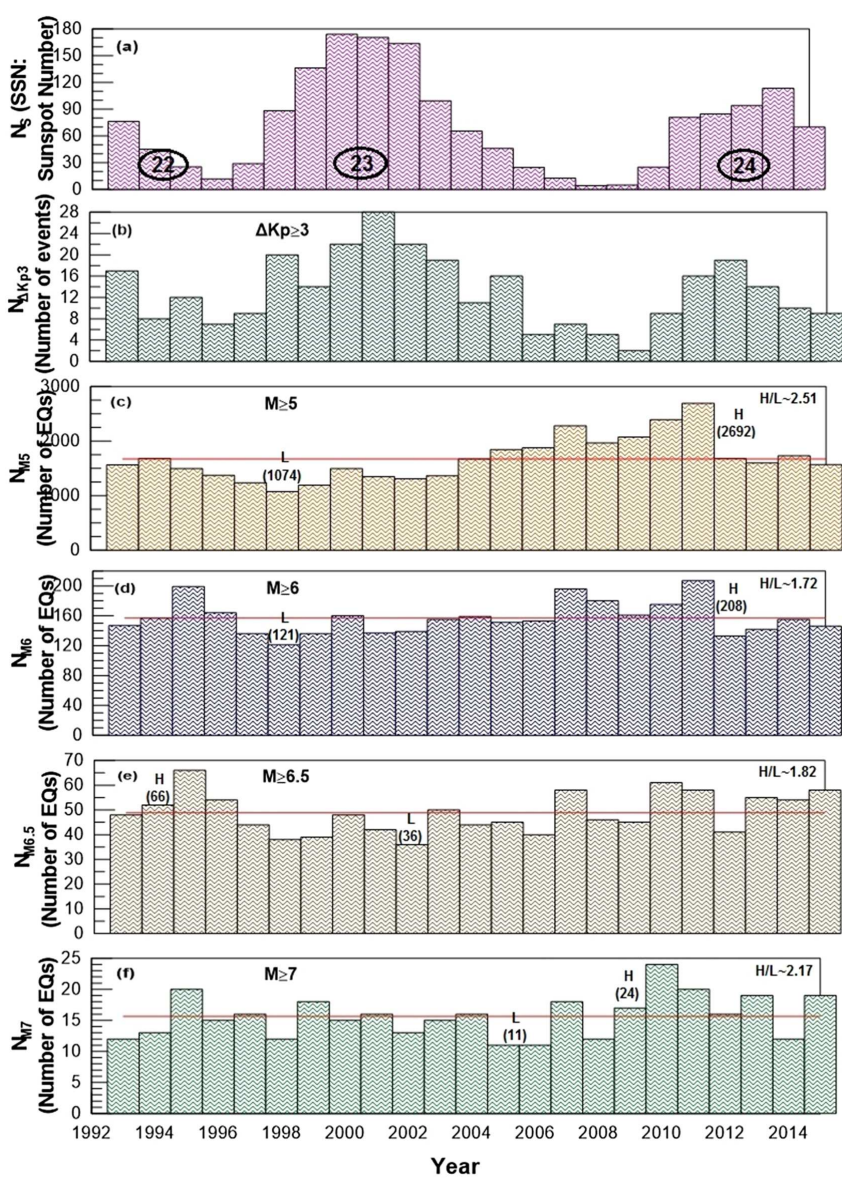

Fig. 6. Solar, large magnetic storm and seismic activity, compared during a period of 22 years (1993-2015). Panel a: The yearly mean sunspot numbers $N_{S}$. Panel b: The yearly number $N_{\Delta K p .3}$ of large magnetic storms identified by very abrupt initial phase $(\Delta K p \geq 3)$ (Panels c-f) and the number of earthquakes per year, with magnitude $M \geq 5$ (NM5), $M \geq 6$ (NM6), $M \geq 6.5$ (NM6.5), $M \geq 7$ (NM7). The red horizontal lines indicate the average values of EQs in each panel. Highest frequencies of EQs of various magnitudes above M5 are seen during the decay phases of SC22 and SC23, as well as during the rising phase of SC24, in agreement with the distribution of the strong/giant EQs of Figure 3; the number enclosed in parentheses indicates the maximum and the minimum number of EQs in each panel. During the maximum phase of SC23, when many CMEs are produced and propagate in the interplanetary space, the EQ frequency shows low values (or even the lowest values during the years 1993-2015). The data suggest a good relation of EQ frequency with the presence of HSSs and not with CMEs.

Here we examine the possible association of EQs of various magnitudes with the solar cycle. In Figure 6 solar, geomagnetic and seismic data are shown for the time interval of 22 years, from 1993 to 2015. In panel (a) we display the yearly mean sunspot number $N_{S}$, which is an index of the solar activity. The most pronounced feature of solar activity is definitely the two sunspot maxima of SC23 and SC24 during the years 2000-2002 and 2013-2014.

In panel (b) we show the number of "abrupt increases" in geomagnetic activity per year, $N_{\triangle K p .3}$, where by the term "abrupt increases" we define here the magnetic 
storms, with increases between successive three-hour values of the $K p$ index higher than three, that is $\Delta K p \geq 3$. Such abrupt jumps typically characterize the CMErelated magnetic storm initial phases, whereas the HSS/CIR- related magnetic storms have more gradual onsets [123].

From the comparison of the two upper panels of Figure 6 (panels a - b) we infer that $N_{\Delta K p .3}$ follows the general distribution type of the sunspot number $N_{S}$ well. When $N_{\Delta K p .3}$ and $N_{S}$ were normalized in order to compare distributions with similar values Spearman correlation coefficient was found to be as high as $r_{S}=0.96$ $(P<0.001)$. The very close positive correlation between $N_{\Delta K p .3}$ and $N_{S}$ is consistent with previous results from many researchers, who have found a close relation between SSN and CME-driven storm activity ([100,102] and references therein). We conclude that the vast majority of the storms with $\Delta K p \geq 3$ (between 1993-2015) were CMEassociated events.

Panels (c)-(f) display the numbers $N_{M .5}, N_{M .6}, N_{M .6 .5}, N_{M .7}$ of earthquakes per year with magnitudes $M \geq 5,6,6.5$, and 7 , respectively. Red lines in panels c-f indicate the average number of earthquakes for each sample $\left(N_{M .5}-N_{M .7}\right)$, during the whole time interval examined (1993-2015).

From a comparison of panels (a), (b) with panels (c)-(f) we infer that the number of earthquakes $N_{M .5}, N_{M .6}, N_{M .6 .5}, N_{M .7}$ show their highest values during the minimum transits from one cycle to the next. In particular, highest numbers of EQs are seen (i) during the decay phase of SC22 (1992-1993: $M \geq 5$ in panel c; 1994-1996: $M \geq 6, M \geq 6.5, M \geq 7$ in panels d-f), (ii) in the decay phase of SC23 (2004-2007: EQs with $M \geq 5$; 2007: EQs with $M \geq 5, M \geq 6, M \geq 6.5, M \geq 7$ ) and (iii) during the ascending phase of SC24 (2010-2011; all panels). On the contrary, the lowest values are seen for a period including the rising and the maximum phase of solar cycle 23 (1998-2002: $M \geq 6, M \geq 6.5, M \geq 7)$.

The deviation of the numbers $N_{M .5}, N_{M .6}, N_{M .6 .5}, N_{M .7}$ is significant throughout the 22 years examined in Figure 6, with highest-to-lowest $\mathrm{H} / \mathrm{L}$ earthquake number ratio ranging between values as high as $1.72-2.51$. These numbers suggest an increase in average by a factor of $\sim 2$ in the number of EQs (panels $d-f$ ) seen in time periods outside the solar maximum phase of SC23 compared to the number of EQs occurring during the maximum phase.

Important to note is that during the period examined in Figure 6 (1992-2015) the distribution of EQs of various magnitudes considered in panels c-f roughly follows the Gutenberg and Richter [46] law. For instance, the maximum yearly value of EQs with magnitudes $M \geq 6$ and $M \geq 5$ are $N_{\mathrm{M} .6}=208$ and $N_{\mathrm{M} .5}=2692$, respectively, which implies more than an order of magnitude ratio $N_{\mathrm{M} .5} / N_{\mathrm{M} .6}$. Furthermore, during the year 2010 (the year with the largest number of $M \geq 7$ EQs), the number of EQs with magnitude $M \geq 5, N_{\mathrm{M} 5}$, reaches a value as high as $\mathrm{N}_{\mathrm{M} 5}=2392$, that is $\sim 2$ orders of magnitude higher than the number of EQs with $M \geq 7: \mathrm{N}_{\mathrm{M} 5} \approx 24 \times 10^{2}$. Such a difference in the number of EQs $N_{\mathrm{M} 5}$ and $N_{\mathrm{M} 7}$ can also be seen during other years with high seismicity throughout the period 1993-2015. However, Shestopalov and Kharin ([107] their Fig. 1) have noted that during some time intervals between 1876-2012 there is a shift of the long term variations of the occurrence frequency of EQs of various magnitudes $(M \geq 6, M \geq 6.8$, and $M \geq 8)$, which suggests that the distribution of EQs of various magnitudes does not always follow the same pattern (an almost Gutenberg and Richter distribution as found in general between 1992-2015).

Another important feature seen in Figure 6 is that a negative correlation pattern between the SSN and the EQ frequency is clearer in the case of EQs of lower magnitudes (panel c and d; $M \geq 5, M \geq 6$ ) than for EQs of greater magnitudes (panel f: $M \geq 7$ ). We believe that this result is due (probably among other reasons) 
to statistical limitations, that is to the small number of EQs with magnitudes $M \geq 7$ occuring within one year $\left(\left\langle N_{M 7}\right\rangle \approx 15 \mathrm{EQs}\right)$.

Solar cycle 24 was very weak (Fig. 3). However, important to note is that the abnormal solar and interplanetary activity of the SC23 deep minimum and of SC24 is associated with an unusually large number of giant $(M \geq 8.3)$ and catastrophic EQs at those times (Fig. 3b), as for instance the M9.1 Sumatra 2004 and Japan 2011 EQs.

A recent study of the geomagnetic storms associated with coronal holes during the time interval 1976-2018 (including SC21-SC24) suggests that the maxima in the speed of the solar wind occurred at the decay phase of all these cycles; namely during the years 1982-1986, 1994-1996, 2003-2009, and 2016-2017 ([131]; Fig. 1). This result is consistent with the high production rate of $\mathrm{CH}$-driven HSSs and the highest frequency of great EQs during the decay phase of solar cycle as causally related events. As in the case of the decay phase of SC23, a high frequency of strong EQs occurred during the rising phase of the weak SC24 (2010-2011), when large areas of coronal hole were observed in EUV AIA/SDO measurements [70].

\subsection{Solar-terrestrial seismic activity relationship during solar cycles 14-24 (1900-2017): Statistical evidence and limitations}

Previous studies have already provided evidence for a solar - terrestrial seismic triggering by studying data during time intervals as long as one century or even more $[81,107,115]$. However, some other authors have disputed any relation between solar and terrestrial seismic activity. The most serious points of the criticism against the solar - terrestrial seismic triggering, according to [69], are: (a) the absence of significant correlation between solar/space and terrestrial parameters and (b) the fact that researchers have claimed either a correlation or anticorrelation between seismic and solar activity. A particular response to the dispute of Love and Thomas [69] is given in the discussion section.

Some of the confusion on the relation between seismic and solar activity comes from the fact that the solar - terrestrial seismic triggering is sensitive to short time (a few months or some weeks) variations of solar activity. The fast wind emanates from coronal holes at high solar latitudes, but near-equatorial holes can form at any time during the eleven-year solar cycle [57]. Also, the $\mathrm{CH}$-driven HSSs/CIRs are mostly observed during the decay phase of the solar cycle, but they often occur during the maximum phase (Hathaway, 2005; [100]). Since, the $\mathrm{CH}$-related HSSs, which seem to be the main space driver of the terrestrial seismicity often lasting for a few weeks, a solar - terrestrial seismicity should be investigated on the basis of at least monthly (and not yearly) averaged data.

Since, we found significant evidence between CH-driven HSSs and terrestrial seismicity in the time interval 1980-2017, we proceed now to examine longer term time series, but by taking into account the fact of the short time variations (a few months) of the solar activity (which reflect the SSN variations).

For this reason, in Figure 7 we compare the occurrence time of giant EQs (normal lines in panels a, b and c) with magnitudes as great as $M \geq 8.5$ with monthly mean SSNs, for the time period from 1900 to 2017 (SC14-SC24). The monthly mean SSN is a tool that can extract important information on the actual solar activity, which is lost in the yearly averaged SSN values. This is evident in Figure 7, where we can see that the four EQs at the maximum phase (SC14, SC17, SC19, and SC 24; purple circles) of the solar cycling, actually occurred at times of a great reduction in the SSN. For instance, the SC14 EQ, with a magnitude M8.8, occurred in January 31, 1906, throughout a reduction in the monthly SSN from 179, in November 1905, to 


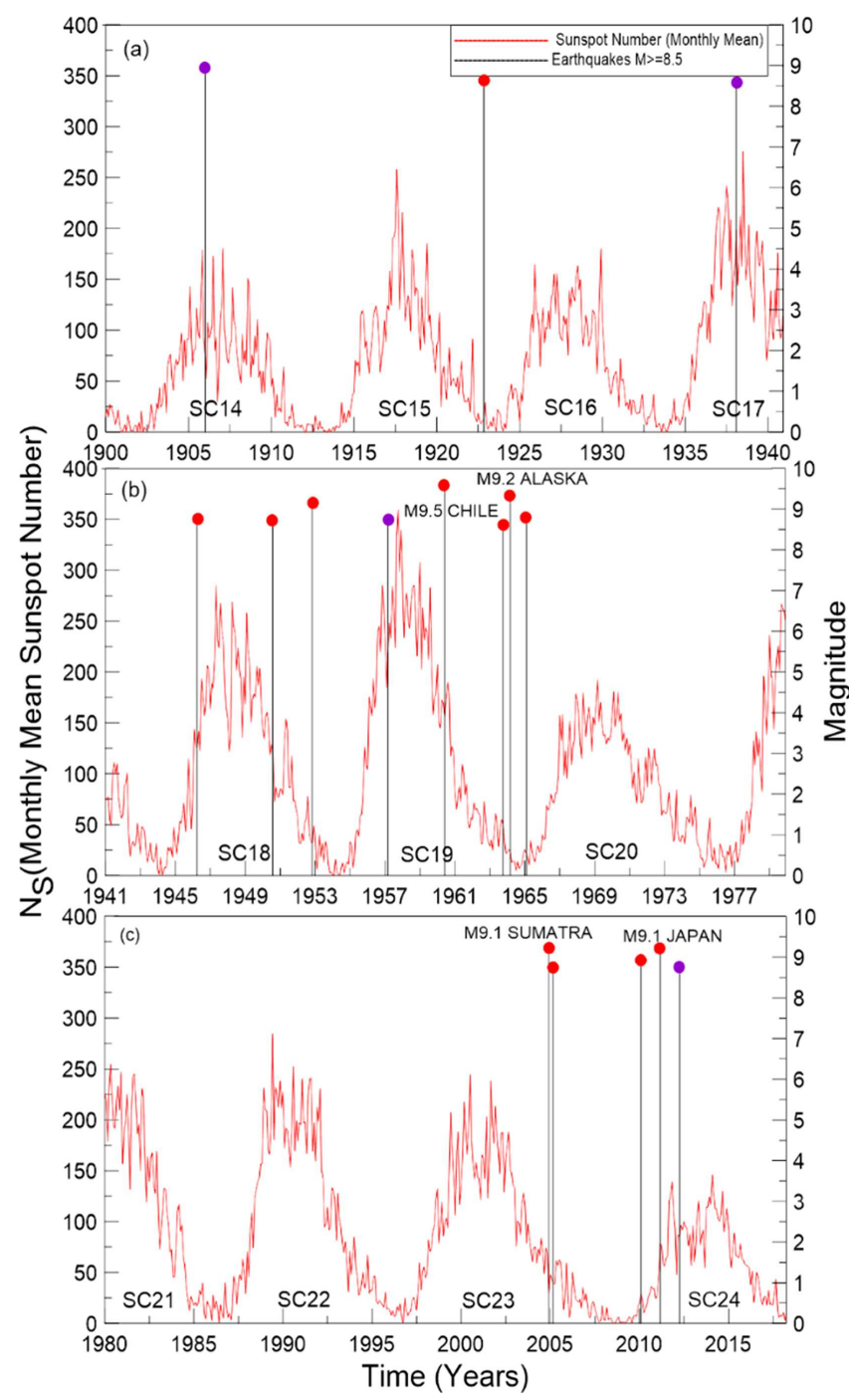

Fig. 7. The occurrence times of giant EQs (normal lines in panels a-c) with magnitudes greater than or equal to $M \geq 8.5$ are compared with the monthly mean SSNs, for the time period from 1900 to 2017 (SC14-SC24). The monthly mean SSN is a tool that can extract important information on the actual solar activity, which is lost in the yearly averaged SSN values. We see that all of the 16 giant $(M \geq 8.5)$ EQs between 1900 and 2017 occurred during the decay, minimum and the rising phase of the solar cycle or at times of a strong reduction, which are to be related with an extension of the conoral hole areas and $\mathrm{CH}$-driven HSSs (see detailed analysis in the text).

a value as low as 52 in February 1906. A similar behavior can be investigated for the M8.6 April 2012 EQ seen at the maximum phase of SC24, but occurred during a dip of the SSN, (from a value of 139 in November 2011 down to 86 in April 2012). A similar profile shows the monthly averaged SSN around the M8.5 1938 EQ and the M8.6 1957 EQ in the maximum phase of the SC17 and SC19, respectively. We point out that SC14 and SC24, when large SSN fluctuations along with big EQs were recorded, were the weakest circles of the period examined in Figure 7. On these lines, 
it is interesting to note that a series of HSSs was recorded by the ACE spacecraft, during an enlarged coronal hole observed by the SDO satellite (Sect. 5.4.2), preceded the giant M9.1 2011 Japan EQ, during the "weak" SC24. This kind of relationship between solar cycle-SSN-HSS-big EQs will be further discussed in the next section (Sect. 5.4).

From the above analysis we infer that solar-terrestrial seismic triggering research based on monthly averaged SSN greatly supports the concept of the solar activity as an agent of terrestrial seismicity. It is interesting to note that the four above mentioned giant EQs occurred during maximum phases of the solar cycle, but at times with monthly (yearly) values ranging within a broad region of SSN (from 76 (90) to 222 (269)), due to the long term variation of the solar activity (see below in the text and Fig. 10). This fact also indicates that the solar - terrestrial seismic triggering concept cannot be well tested by studies comparing seismic activity with the yearly $\mathrm{SSN}$, as the two phenomena were related by a permanent function, independently of the long-term (several decades) variations of the SSN (see also Sect. 6.2.2). For instance, since the amplitudes of two solar cycles were 76 and 222 and giant $(M \geq 8.5)$ EQs occurred in both cases, we infer that the yearly SSN could not be a precise index predicting high seismicity.

Moreover, we can see that most (twelve out of sixteen) of the giant EQs shown in Figure 7 (indicated by a red solid circle) occurred during the decay (8 EQs), the minimum (1 EQ), and the rising (3 EQs) phase of the solar cycle. Three giant extremely catastrophic EQs were occurred during the decay phase (Chile M9.5 1960 EQ, Alaska M9.2 1964 EQ, Sumatra M9.1 2004 EQ) and one EQ (Japan M9.1, 2011) during the onset phase of the "strange" SC24 (Sect. 6.2.2).

We infer, therefore, that all of the 16 giant $(M \geq 8.5)$ EQs between 1900-2017 occurred during the decay, minimum and the rising phase of the solar cycle or during the maximum phase, but at times of a strong reduction of the SSNs, which is known to be related with an extension of the coronal hole areas and CH-driven HSSs [57, 100,123]; this result is consistent with the results found by studying big EQs for the shorter period between 1980-2017 (Fig. 3).

In Figure 8 we present data in order to further elaborate both the solar-terrestrial seismic activity relationship and the limitations posed by some statistical studies. For this reason, we compare the yearly values of SSN with both the occurrence frequency of large $(M \geq 7)$ EQs as well as the global seismic energy, during the same time interval as in Figure 7 (1900-2017). The released energy was calculated using the Richter scale $E=10^{(4.8+1.5 \mathrm{M})}$, where $E$ is the released energy and $M$ is the EQ magnitude [46].

Although in most statistical studies on the solar-terrestrial seismic relationship the occurrence frequency of distinct large EQs has been examined, the hypothesis of the solar electromagnetic energy transfer as a major agent of the terrestrial seismicity imposes the examination of the global seismic energy output as well.

Panels c-e of Figure 8 show the yearly averaged values of the $\operatorname{SSN} N_{s}$, the number of $M \geq 7$ EQs $N_{M 7}$ and the seismic energy $E_{S}$ of $M \geq 5.5$ EQs, respectively. The normal dashed lines (numbered 1-21) indicate the peaks in seismic energy $E_{S}$, which are related with EQ occurrence times at the decay, minimum and the rising phase of a solar cycle. From a comparison of the $E_{S}$ peaks (panel e) with the $N_{s}$, (panel c) we can make several comments.

First, we see that the vast majority of the seismic energy $E_{S}$ peaks (21 out of a total of 27) were observed during the decay (14), the onset (5) and the minimum (2) phase of the solar cycle, whereas only 6 peaks were related with the maximum phase. As "maximum phase" we considered here the three-year intervals with highest SSN $N_{s}$ in each solar cycle, with an exception for the case of SC15, due to its spiky form. 


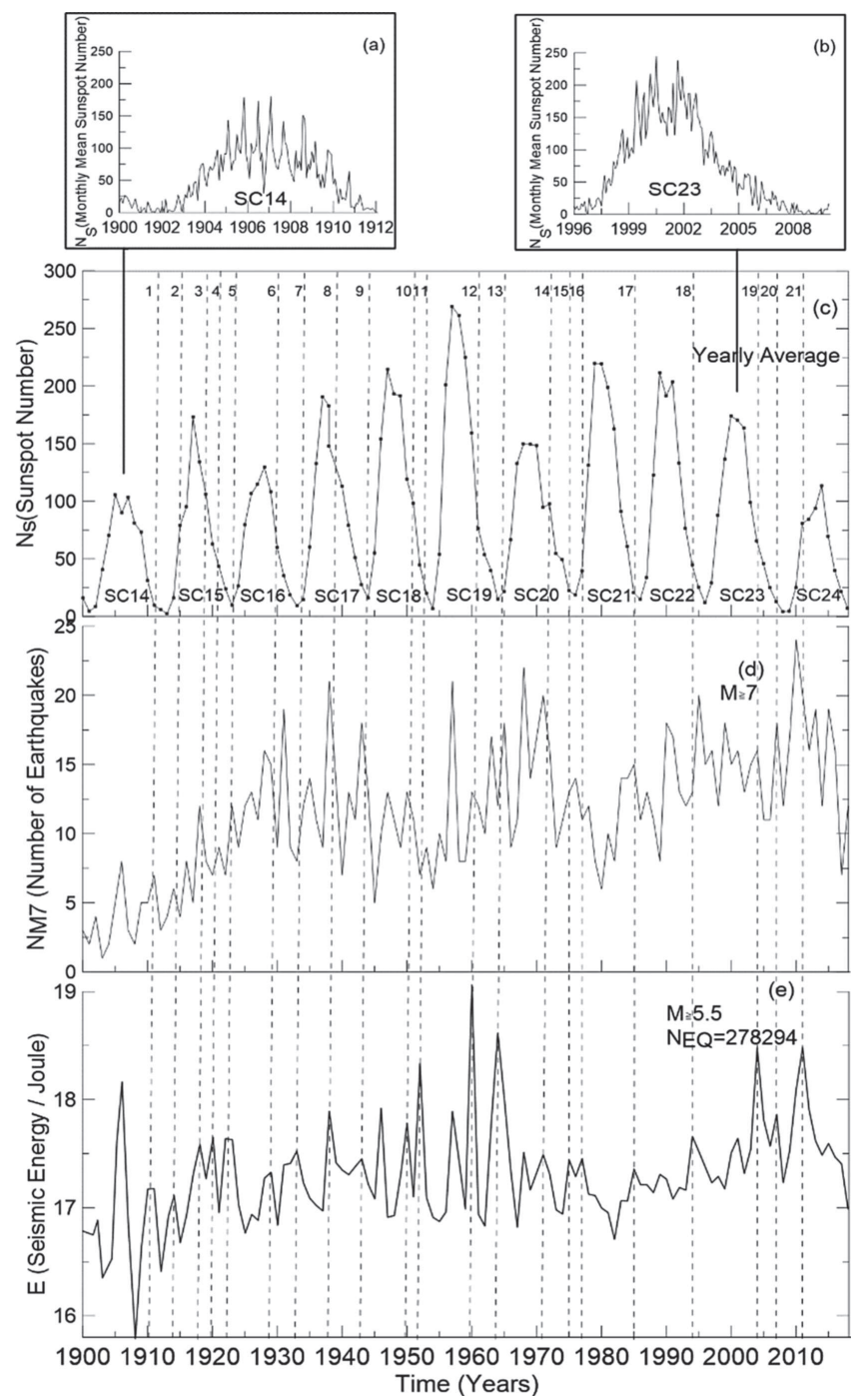

Fig. 8. Panels c, d and e show the yearly averaged values of the SSN Ns, the number of $M \geq 7 \mathrm{EQs} N_{M 7}$ and the seismic energy ES of $M \geq 5.5 \mathrm{EQs}$, respectively. The normal dashed lines (numbered 1-21) indicate the peaks in seismic energy E, which were related with EQ occurrence times at the decay, minimum and the rising phase of the solar cycle. We see that the vast majority of the seismic energy ES peaks (panel e) were observed during the decay, the rising and the minimum phase of the solar cycle (panel c). The number of $M \geq 7$ EQs $N_{M 7}$ in general shows a more complex profile (see discussion in the text).

Furthermore, we can see that all of the five most intense $E_{S}$ peaks were observed during the decay $(\# 11,12,13,19)$ and the rising (\#21) phase.

The peaks at the maximum of the SC14 and the SC23 were observed during dips in the monthly number of the SSN (panels a and b in Fig. 8). Furthermore, we see peaks at the maximum of the SC19 and SC20, but their maximum values are low compared to the close peaks during decay phase of SC19, $E_{S}$ peak \#12, and the rising phase of $\mathrm{SC} 20, E_{S}$ peak \#13. 

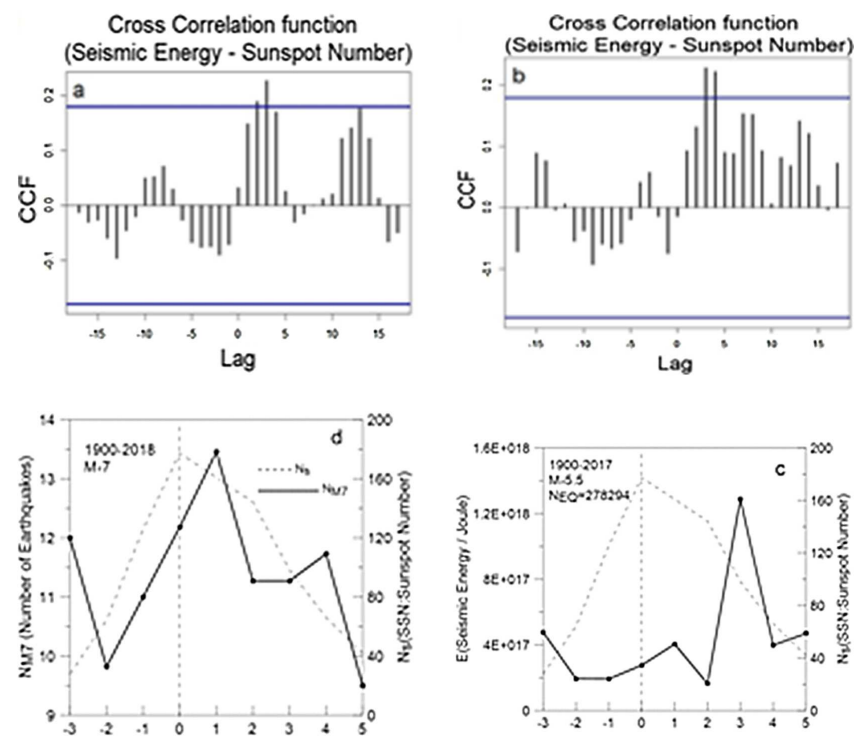

Fig. 9. (Panel a) The estimated cross-correlation coefficient (CCF) between the yearly numbers of the seismic energy ES and the Sunspot Number NS shown in Figures 8c and 8e, for the time interval 1900-2017. It is obvious that there is a delay of three years in the seismic energy output $E_{S}$ compared to the year of the maximum SSN, which is significant at a level $P<0.05$ (horizontal blue lines). (b) As in panel a, but when the seasonality was subtracted in the SSN time series. (c) Results from the super epoch analysis' method applied for the seismic energy $E_{S}$. This figure also suggests that the maximum seismic energy is released during the third year after the sunspot maximum (normal line at 0). (d) As in panel c, but for the number of earthquakes of Figure 8d, with magnitudes $M \geq 7$ (see discussion in the text).

Since from Figures 3, 7 and 8 we saw a preferential appearance of EQs during the decay phase of the solar cycle, we performed statistical tests to investigate the significance of this phenomenon. For this reason, in Figure 9a we present the estimate of the cross-correlation coefficient (CCF) between the two time series of the yearly numbers of the seismic energy $E_{S}$ and the Sunspot Number $N_{S}$ shown in Figures 8c and 8e, between 1900 and 2017. The solid line in the middle corresponds to the hypothesis that the two time series are independent, while the dashed lines correspond to the $95 \%$ confidence limits under the hypothesis that the two time series are white noise. Values of the estimate outside the confidence limits indicate deviations of the hypothesis of independence. In this case, it is obvious that there is a delay of three years between the two time series. That is, there is a delay of three years of the seismic energy output $E_{S}$ compared to the year of the maximum SSN. From the same figure the solar cycle ( $\sim 11$ year) variation of the data is also evident.

Figure $9 \mathrm{~b}$ presents the estimate of the CCF between the two time series of seismic energy $E_{S}$ and the Sunspot Number $N_{S}$ when the (solar cycle) seasonality in the SSN time series was subtracted. This has been necessary, because the presence of the seasonality can produce problems [23]. According to this figure there is also a delay of 3-4 years between the two time series, which is significant at a level $P<0.05$.

Furthermore, since in previous statistical studies, the superposed epoch analysis (SEA) method was used to check the possible connection between solar activity and seismic activity, in Figure 9c we present the results from the SEA applied for the same data as in the evaluation of CCF. This figure also suggests that the maximum seismic 
energy is released during the third year after the sunspot maximum, in agreement with the CCF and the results presented by Odintsov et al. ([81]; their Fig. 6). A Student's $t$-test for the peak energy during the third year after the sunspot maximum $E(+3)$ compared to the energy during the sunspot maximum $E(0)$ suggests a weak statistical significance, at a level $P \leq 0.1$.

Figure 8d presents the yearly number of EQs $N_{M 7}$ with magnitude $M \geq 7$ for the time interval 1900-2017. A comparison of the $N_{M 7}$ and $E_{S}$ time series suggests that $N_{M 7}$ shows a much more complex profile than the global energy output $E$. The most characteristic features of the number of EQs with magnitude $M \geq 7$ are the following: (a) intensive seismic activity, as indicated by $N_{M 7}$, was observed during low solar activity, in particular during SC16-SC17, SC20, SC22-SC24 and low $N_{M 7}$ activity was observed during large amplitude solar cycles, as for instance, during SC18-SC19, SC21; in this case there is an evident negative correlation between $N_{S}-N_{M 7}$, (b) peaks in the number of EQs $N_{M 7}$ are seen around all of the 21 peaks of seismic energy output $E_{S}$ (mostly during rising-decay-minimum phases, but also during the maxima of SSN).

The SEA method was used for $N_{M 7}$, as in the case of $E_{S}$ (Fig. 9d), which shows two peaks with a delay of one year and four years compared to the year of the sunspot maximum; these peaks are not significant enough when the Student's $t$-test is applied.

In Figure 10, the time series of the yearly average sunspot numbers $N_{S}$ (panel a) and the annual number of earthquakes with $M \geq 7 N_{E}$ (panel b) are presented from 1900 to 2017. In both data sets we present the corresponding trends (red lines), which were created by using a smoothing spline [44]. The trends play a significant role as they show the change of the mean values through time. Visually, it seems that the trends have a clear negative correlation between the years 1930-2010: when the value of the SSN increases then the number of EQs decreases and vice versa.

The Spearman correlation coefficient $\left(r_{S}\right)$ of the two data sets of Figure 10 was found to be equal to -0.42 , with a p-value $<0.0001$. This extremely low p-value of the correlation coefficient suggests a very high significant negative correlation between the long term variation of the SSN and the number of earthquakes with $M \geq 7$, between the years $1930-2010$. The two shaded areas at the beginning and at the end of the $N_{S}$ and $N_{E}$ curves are parts of the whole time interval examined in Figure 8, and they are not included in the calculation of the $r_{S}$, since their inclusion destroys the negative correlation between the two magnitudes $N_{S}$ and $N_{E}$; this is a fact we see when the sunspot number $N_{S}$ takes very low values (Sect. 5.2).

In conclusion, from the examination of monthly, yearly and long term variations of the SSN and seismic activity between 1900 and 2017, we infer that the giant EQs and the global energy output from all EQs with magnitudes $M \geq 5.5$ are preferentially related with the decay phase of the solar cycle. Furthermore, the statistical tests confirm a significant negative correlation between the sunspot number and the global seismic energy release, except for time periods with very low levels of SSN.

The number of EQs with magnitudes $M \geq 7$ shown in Figure $8 \mathrm{~d}$ is not in good agreement with the pattern of a negative correlation between seismicity and the SSN (Fig. 10d). This fact may be due to: (a) the statistical limitations (small number) of the great EQs with magnitudes $\sim 7<M<\sim 8$, occurring within one year, (b) the appearance of CH-driven HSSs throughout the whole solar cycle, (c) the direct association of great EQs with CH-driven HSSs and not with the SSN (different solar activity has been confirmed throughout the solar cycles under the same SSN), (d) the decrease or absence of strong CH-driven HSSs during times of very low SSNs as in the case of 2008-2009 and the Maunder (1645-1720) and the Dalton (1790-1820) minima and the destruction of the negative correlation between solar and seismic activity, (e) the magnitude (time)-dependent long term variations of EQ occurrence 


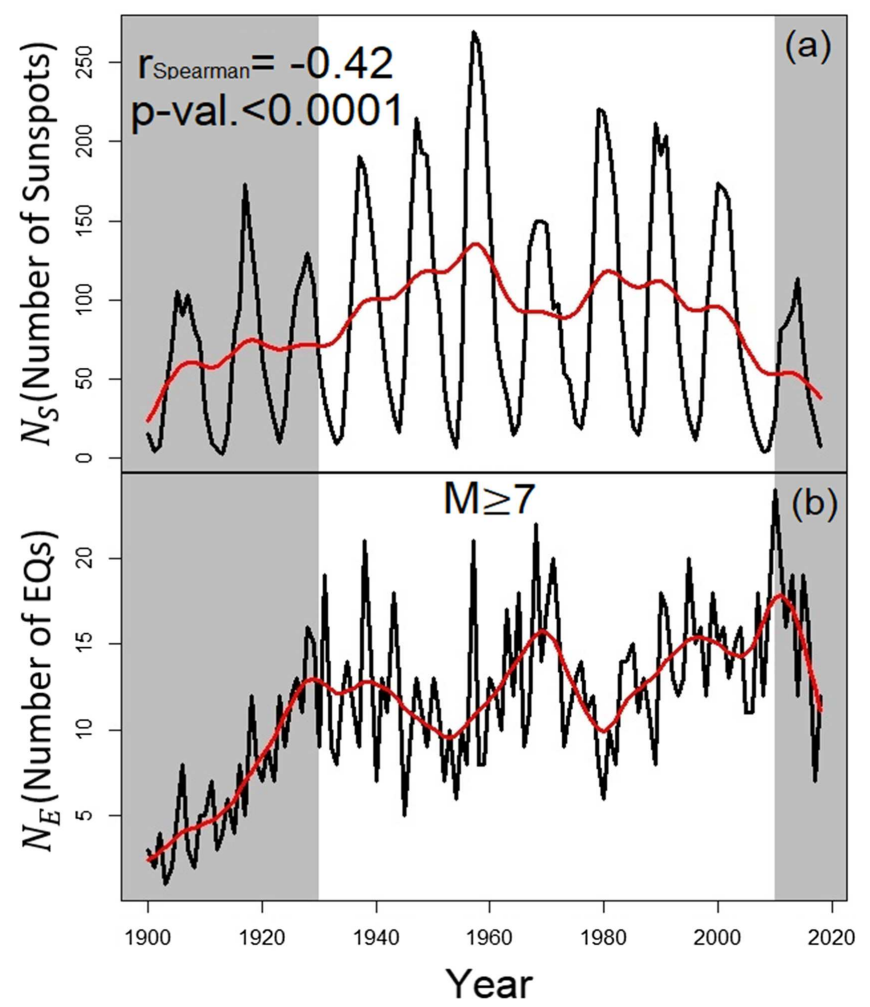

Fig. 10. Time series of the yearly average sunspot numbers $N_{S}$ (panel a) and the annual number of earthquakes $N_{E}$ with $M \geq 7$ (panel b) from 1900 to 2017. The corresponding trends (red lines) obtained by using a smoothing spline are also shown. The trends show a very significant negative correlation $\left(r_{S}=-0.42, p<0.0001\right)$ between the long term variation of the SSN and the number of earthquakes with $M \geq 7$ during the period 19302010, with, in general, high amplitude solar cycles. The two shaded areas at the beginning and at the end are parts of the whole time interval, but are not included in the calculation of the $r_{S}$, since their inclusion destruction the negative correlation between the two magnitudes $N_{S}$ and $N_{E}$, which is a fact we see when the sunspot number $N_{S}$ takes very low values (Sect. 5.2).

frequency [107], and (f) a possible contribution of CME-related effects mostly during the solar maximum.

We infer that the giant EQs and the global energy release, which reflect large spatiotemporal scale solar-terrestrial processes, follow a negative correlation pattern with the yearly SSN well, except for time intervals with very low levels of SSN.

\subsection{Coronal holes solar wind streams and giant earthquakes (1980-2017)}

\subsubsection{High speed streams and earthquakes}

The plasma flow from the solar corona is non-uniform in both time and space. The emanating solar wind from the solar corona shows a latitudinal dependence, with the highest values $(\sim 500-800 \mathrm{~km} / \mathrm{s})$ over the poles and lower values $(\sim 300-400 \mathrm{~km} / \mathrm{s})$ towards the Sun's equator, when it arrives near Earth. The fast wind emanates from coronal holes, dark regions in the corona, where the magnetic field is "open", 
Table 3. Data for the giant $M \geq 8.3$ earthquakes which occurred during the period 19802018 (January).

\begin{tabular}{|c|c|c|c|c|c|c|c|}
\hline $\begin{array}{l}\text { EQs } \\
(\#)\end{array}$ & Country, Region & Date & Time & $M$ & $\begin{array}{l}\text { Longit. } \\
\text { (deg) }\end{array}$ & $\begin{array}{l}\text { Latit. } \\
\text { (deg) }\end{array}$ & $\begin{array}{l}\text { Depth } \\
(\mathrm{km})\end{array}$ \\
\hline 1 & Russia Kuril Islands & $4 / 10 / 1994$ & $13: 23$ & 8.3 & 147.3 & 43.8 & 14 \\
\hline 2 & $\begin{array}{l}\text { Peru Near the coast of south } \\
\text { era Peru }\end{array}$ & $23 / 6 / 2001$ & 20:33 & 8.4 & -73.6 & 16.3 & 33 \\
\hline 3 & Japan Hokkaido Japan rezion & $25 / 9 / 2003$ & 19:50 & 8.3 & 143.9 & 41.8 & 27 \\
\hline 4 & Indonesia Andaman & $26 / 12 / 2004$ & 00:59 & 9.1 & 96.0 & 3.3 & 30 \\
\hline 5 & Indonesia Northern Sumatra & $28 / 3 / 2005$ & 16:09 & 8.6 & 97.1 & 2.1 & 30 \\
\hline 6 & Russia Kuril Islands & $15 / 11 / 2006$ & $11: 14$ & 8.3 & 153.3 & 46.6 & 10 \\
\hline 7 & Indonesia Southern Sumatra & $12 / 9 / 2007$ & $11: 10$ & 8.4 & 101.4 & -4.4 & 34 \\
\hline 8 & Chile Offshore Bio-Bio & $27 / 2 / 2010$ & 06:34 & 8.8 & -72.9 & -36.1 & 22.9 \\
\hline 9 & $\begin{array}{l}\text { Japan Near the east coast of } \\
\text { Honshu }\end{array}$ & $11 / 3 / 2011$ & 05:46 & 9.1 & 142.4 & 38.3 & 29 \\
\hline 10 & $\begin{array}{l}\text { Indonesia Off the west coast } \\
\text { of N. Sumatra }\end{array}$ & $11 / 4 / 2012$ & 08:38 & 8.6 & 93.1 & 2.3 & 20 \\
\hline 11 & Japan-Russia Sea of Okhotsk & $24 / 5 / 2013$ & 05:44 & 8.3 & 153.2 & 54.9 & 598.1 \\
\hline 12 & Chile & $16 / 9 / 2015$ & $22: 54$ & 8.3 & -71.7 & -31.6 & 22.44 \\
\hline
\end{tabular}

streaming into interplanetary space mostly during the decay phase of a solar cycle, when polar $\mathrm{CHs}$ extend towards the solar equator. In some cases the $\mathrm{CH}$-driven HSS forms a corotating interaction region (CIR), which is bounded by two MHD shocks: the forward shock at the front of the CIR and the following reverse shock at the end of the CIR. Coronal holes also appear at mid-to-low solar latitudes, either as discrete openings or as a narrow, finger-like extension from a polar coronal hole, during solar maximum phase.

What is the most evident result of the present study is the fact that most of the giant $(M \geq 8.3)$ EQs, but also smaller EQs with magnitudes $M \geq 5$, during the four recent solar cycles (Figs. 3, 6-8), occurred in the decay phase and close to solar minimum; these are times when near-equatorial CHs and HSSs are also often observed $[57,100,102,103]$.

In order to further check the relation between $\mathrm{CH}-\mathrm{HSS} / \mathrm{CIR}$ - EQs, we examined the space weather before the twelve giant $(M \geq 8.3)$ EQs of Figure 3b. Table 3 summarizes the main features of the giant 12 EQs of Figure 3b. A detailed study of the ACE magnetic field, plasma and energetic particle observations from three different instrumentations, ACE/MAG (http://www.ssg.sr.unh.edu/mag/ACE.html), bulk plasma data instrument (http://www.srl.caltech.edu/ACE) and EPAM (http:// sd-www.jhuapl.edu/ACE/EPAM) has been done for each one of the EQs numbered 2-12 in Table 3 (the EQ \#1, Russia 4.10.194, occurred before the launch of the ACE spacecraft; see Sect. 4), for more than one month before the EQ occurrence. We came to the conclusion that HSSs and/or CIRs preceded the occurrence of all the 11 giant EQs for long time periods of several weeks to a few months.

A representative example of ACE observations is shown in Figure 11. In this figure we display solar wind data from the ACE spacecraft from August 22, 2015 to September 16, 2015, a total of 25 days, which is about the time of one solar rotation ( $\sim 27$ days). The panels, from top to bottom, display the magnetic field magnitude $B$, the polar coordinates of the field $\varphi$ and $\theta$, the uncertainty (rms) of $B$, the radial component of the solar wind velocity $V r$ and the density of the proton plasma $N p$. In Figure 11 we can see certain HSSs, which are marked as HSS-1, HSS-2, HSS-3 and HSS-4. All of these HSSs follow the typical profile of CH-related structures (Fig. 2), where in the beginning there exists an increase in the values of $B$ and $N p$, and then an 
increase in the solar wind speed $V r$; the events are also characterized by strong large amplitude Alfvén wave activity (mostly variations in $B$ field latitudinal component $\varphi$ suggesting a $B_{z}$, component highly fluctuating between high positive and negative values; second panel from the top). Between HSS-3 and HSS-4, there exists a CIR (7-12.9.2015). The forward (FS) and the reverse shock (RS) are also seen and are marked at the edges of the CIR.

The M8.3 Chile EQ occurred on September 16th, 2015 at the end of the relatively small HSS-4 ( $\sim 2$ days from its beginning), about six days after the reverse shock (RS) of the CIR, and at the end of a period beginning on August 23 (a total of 25 days) with almost continuous large amplitude Alfvén wave activity (except for a period downstream of the FS of the CIR), in agreement with Tsurutani et al. [123], who have described this kind of Alfvén waves as lasting from days to several weeks in association with the HSS. It is worth noting that this sequence of HSSs-CIR, which occurred before the giant and catastrophic 2005 Chile earthquake, resembles the sequence of HSSs seen before the Sumatra-Andaman M9.1 EQ, in December 2004, which has been described in detail by Anagnostopoulos and Papandreou [5].

\subsubsection{Large solar corona holes before giant EQs}

The CH-driven HSSs have very different features to CME-associated HSSs, and therefore, we could define the type of the HSSs observed before the 11 giant $(M \geq 8.3)$ EQs of Figure 3b (Tab. 3, EQs \#2-12; Sect. 5.4.1). However, we can further directly confirm the origin of the HSSs based on solar images obtained during the life of the SDO satellite (February 11, 2010 - today). Indeed, we examined solar images from the outer layer of the Sun's atmosphere, the corona, obtained in the passband of $193 \AA$ by the telescope AIA, during the life of the SDO satellite, for the \#7-12 EQs of Table 3, and we saw the presence of large coronal holes.

In Figure 12 we show representative images of the solar corona $(\sim 2$ days $)$ before three giant and catastrophic earthquakes of Table 3 (Fig. 3b): Japan 2011 (M9.1), Sumatra 2012 (M8.6) and Chile 2015 (M8.3) EQs. The large polar (Figs. 12a and 12c) or near equatorial coronal holes (Fig. 12b) are seen in the three images as dark regions. The large coronal hole seen in Figure 12c is related with the HSS preceding the Chile 2005 EQ examined in Figure 11.

Further examination of some recent catastrophic EQs confirms a similar preseismic presence of solar coronal holes, as for example, in the cases of the Nepal 2015 (M7.8) and Kumamoto and Japan 2016 (M7) earthquakes.

The comparison of the representative solar images with the observation of HSSs/CIRs supports the hypothesis of successive HSSs observed before the giant EQs (Tab. 3) as driven by coronal holes.

\subsection{The Solar rotation period of 27 days in seismological data}

Given that there is good evidence for a "preference" of enhanced seismic activity during the decay phase of solar cycle, when well structured CH-related HSSs/CIRs rotate with approximately the mean solar rotation period (SRP) of $\sim 27$ days, an interesting idea was to examine the possible appearance of the solar rotation period in seismological data. During the decay and the minimum phase of the solar cycles, HSSs/CIRs are usually observed quasi-periodically, with the solar rotation period of $\sim 27$ days, for several months $[57,100,123]$. If the repetitive impact of the $\mathrm{CH}$-related HSSs / CIRs on the Earth's magnetosphere was a significant factor in provoking EQ occurrence, the periodicity of $\sim 27$ days might be detectable in seismological data during the decay phase of the solar cycle. Therefore, we decided to examine 

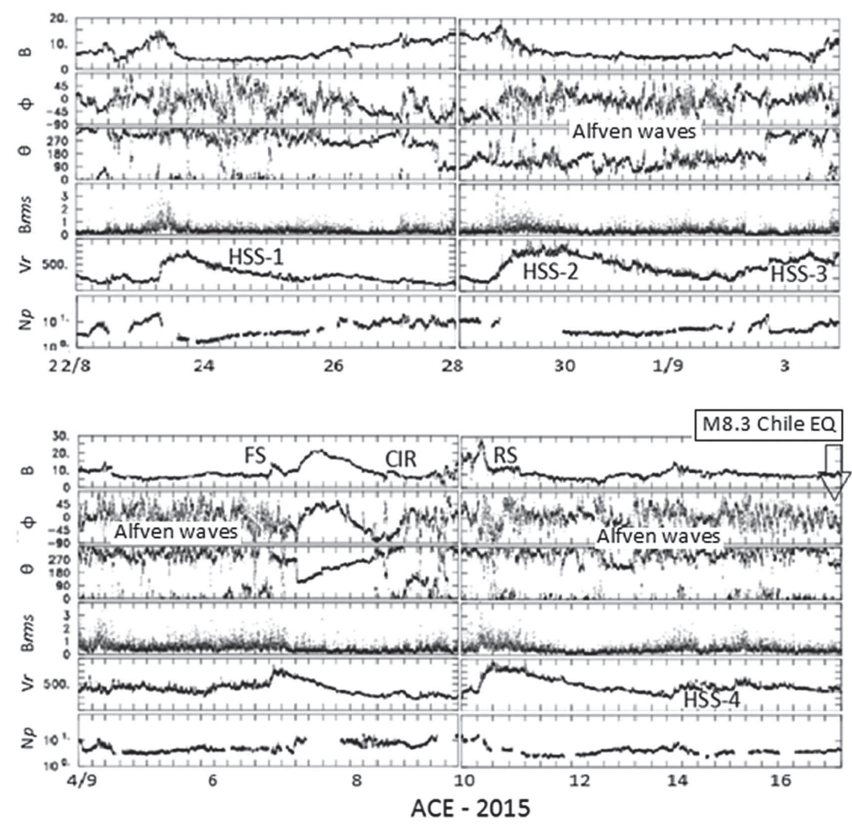

Fig. 11. Representative long time period with almost continuous presence of high amplitude Alfvén waves before the giant (M8.3) Chile EQ, which occurred on September 16th, 2015. The figure displays solar wind data from the ACE spacecraft from 22.9.2015 to 16.9.2015 (a total of 28 days). The panels, from top the bottom, display the magnetic field magnitude $B$, the polar coordinates of the field $\varphi$ and $\theta$, the uncertainty $B_{r m s}$, the radial component of the solar wind velocity $V_{r}$ and the density of the proton plasma $N_{p}$. Several CH-driven HSSs marked as HSS-1, HSS-2, HSS-3 and HSS-4 and one CIR cover the period with almost continuous Alfvén wave activity (variations mostly in $B$ field latitudinal component $\varphi$ in panel b). The forward (FS) and the reverse shock (RS) are marked at the edges of the CIR. The 2015 M8.3 Chile EQ occurred at the end of a time period (1 month) with almost continuous Alfvénic activity. The pre-EQ space conditions resemble the sequence of HSSs seen before the Sumatra-Andaman M9.1 EQ, in December 2004 (Anagnostopoulos and Papandreou, 2012).

whether such a periodic pattern is observable in global energy seismicity. If such a $\sim 27$ day periodic pattern was evident in seismological data, the $\mathrm{CH}$-related 27-day repetitive interplanetary structures should be considered a serious candidate agent for EQ triggering. Moreover, if the 27-day periodicity is observable in the decay phase of the solar cycle and the Sun is confirmed as an agent of EQs, the 27-hour periodicity might not be evident during extended times of an active Sun, as for instance during the maximum phase, when the simple spiral arm geometry of the IMFs are often disturbed from non-periodic phenomena like the CMEs.

Since from the studies presented above we inferred that the $M \geq 5$ EQ occurrence is sensitive to CH-driven HSSs / CIRs, we decided to first check the possible presence of 27-day periodicity for the great EQs with magnitudes $M \geq 6$. Since these EQs may be separated by a long time interval of several days, a spectral analysis of time series of data averaged over several days has to be performed. In this case a $\sim 27$-hour interval should be composed of a few points (for instance, 3 points if a 9-day analysis was decided) and the 27-day periodicity could not be determined. For this reason, we used an appropriate method for the evaluation of a power spectrum by considering our data as a point process. The whole interval (2004-2010) was divided 


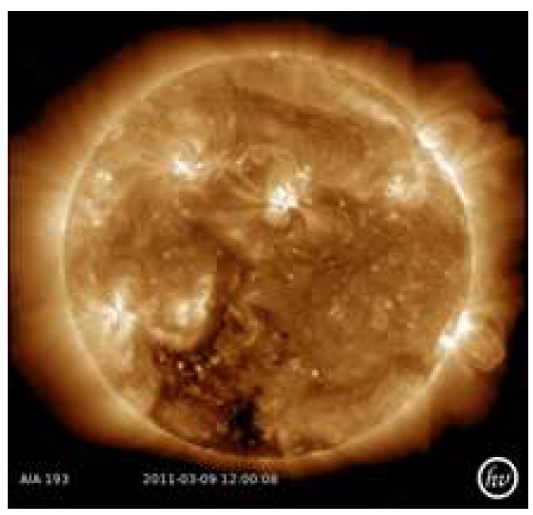

(a)

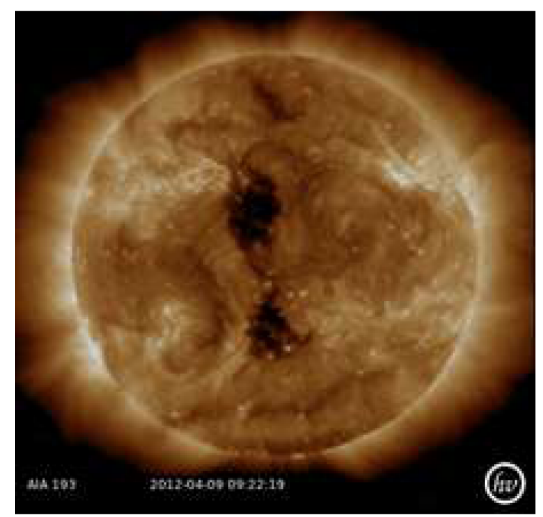

(b)

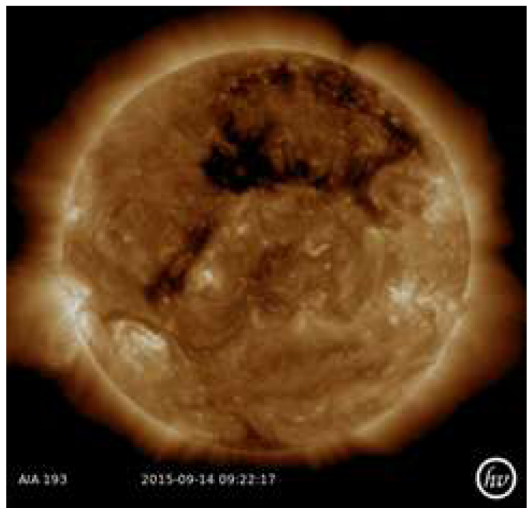

(c)

Fig. 12. Representative images with coronal holes before giant earthquakes. This figure shows images from the outer layer of the Sun's atmosphere, the corona, obtained by the telescope/passband of $193 \AA$ of the Atmospheric Imaging Assembly (AIA) onboard the Solar Dynamics Observatory (SDO) satellite. The images a-c were observed approximately two days before the giant and catastrophic Japan 2011 (M9.1), Sumatra 2012 (M8.6) and Chile 2015 (M8.3) earthquakes. The large polar or near equatorial coronal holes are seen in the three images as dark regions. (a) SDO, 9.3.2011 (Japan M9.1 EQ, 10.11.2011), (b) SDO, 9.4.2012 (Sumatra M8.6 EQ, 1.4.2012), (c) SDO, 14.09.2015 (Chile M8.3 EQ, 16.9.2015). 
into small intervals of four hours each, in order to construct the point process, which consists of binary data ( 0 and 1$)$, where 0 means that there is not an energy release from $M \geq 6$ EQs in the 4-hour interval considered and 1 means that there is an energy release from $M \geq 6$ EQs in the corresponding 4-hour interval. The short time of four hours was selected in order to avoid the occurrence of more than one $M \geq 6$ earthquake within the interval selected as much as possible (Appendix A about the estimate of the auto intensity for a point process). Furthermore, the 2004-2010 was selected, apart from the rich presence of HSSs/CIRs, in view of an additional reason; this is the life time of the first dedicated satellite (DEMETER: Detection of Electro-Magnetic Emissions from Earthquake and Volcanoes) for observing EQ electromagnetic precursory signals. Thus, if a periodic signal at $\sim 27$ days could be recognized in Earth's global seismicity, the $\sim 27$-day solar rotation periodicity (and therefore the CH-driven HSSs) might have influenced the DEMETER data as well.

Figure 13a shows the logarithm to base 10 of the estimates of the spectral density (blue lines) of the seismic energy release (four hours average) of EQs with magnitude $M \geq 6$, during years 2004-2010, which is a period including the decay phase of solar cycle \#23 and the following deep solar minimum. Figure 13b shows the same kind of data, but albeit for the years 1999-2002, that is the maximum phase of SC23, and provides the possibility of studying possible differences of the $\sim 27$-day quasi-periodic effects of HSSs throughout the solar cycle.

The data shown in Figure 13 have been obtained by smoothing the periodogram of the point process by using 17 adjacent ordinates [21]. The red lines indicate approximate $95 \%$ confidence limits around the logarithm to base 10 of the power spectrum estimates of a Poisson process, with the same number of events as those included in the two time series [104]. The number of $M \geq 6$ EQs occurring during the first time interval (decay + minimum) was $N_{E Q}=1175$ and during the second one (maximum) was $N_{E Q}=572$.

From Figure 13a we see that there is a clear peak (denoted by a black dot) outside the $95 \%$ confidence limits. This peak is significant at $5 \%$ level and indicates the presence of an important periodicity at 26.6 days. It is very close to the solar rotational period of $\sim 27$ days and is consistent with the hypothesis of CIRs/HSSs as a significant factor triggering $M \geq 6$ earthquakes during the decay and the minimum phase of SC23. The fact that the spectral peak at $\sim 27$-day is significant confirmed by an evaluation of the autointensity function ([20]; more information about the autointensity function and its interpretation are given in Appendix A and in Fig. 15). This 27-day periodicity in seismological data is a novel result in seismological studies and strongly supports the concept that the $\mathrm{CH}$-driven corotating HSSs, is an important agent in provoking great $(M \geq 6)$ EQs.

A significant peak at $5 \%$ level is also evident in Figure 13b, during the maximum phase of solar cycle \#23 (years 1999-2002), but at a period of 31 days, that is a time interval different to the solar rotational period of 27 days. The comparison of the above results suggests that the peak seen in the logarithm of the estimate of the spectral density in panel a (during the decay phase as well as the following deep solar minimum of solar cycle \#23) is much closer to the solar rotational period of $\sim 27$ days than the peak seen in panel b (during the solar maximum of SC23). The deviation of the spectral peak from the 27-day SRP during the maximum phase is a rather reasonable result. It is well known that during the decay phase of the solar cycle, coronal hole structure on the solar surface is long-lived, and as a result 27-day-recurring HSS driven storms are often found [18,100]. On the contrary, during the maximum phase of the solar cycle, the active Sun strongly affects the solar wind geometry and the CME effects predominate on the interplanetary space and the Earth's magnetosphere/ionosphere. At those times, the usual production of CMEs disturbs the 


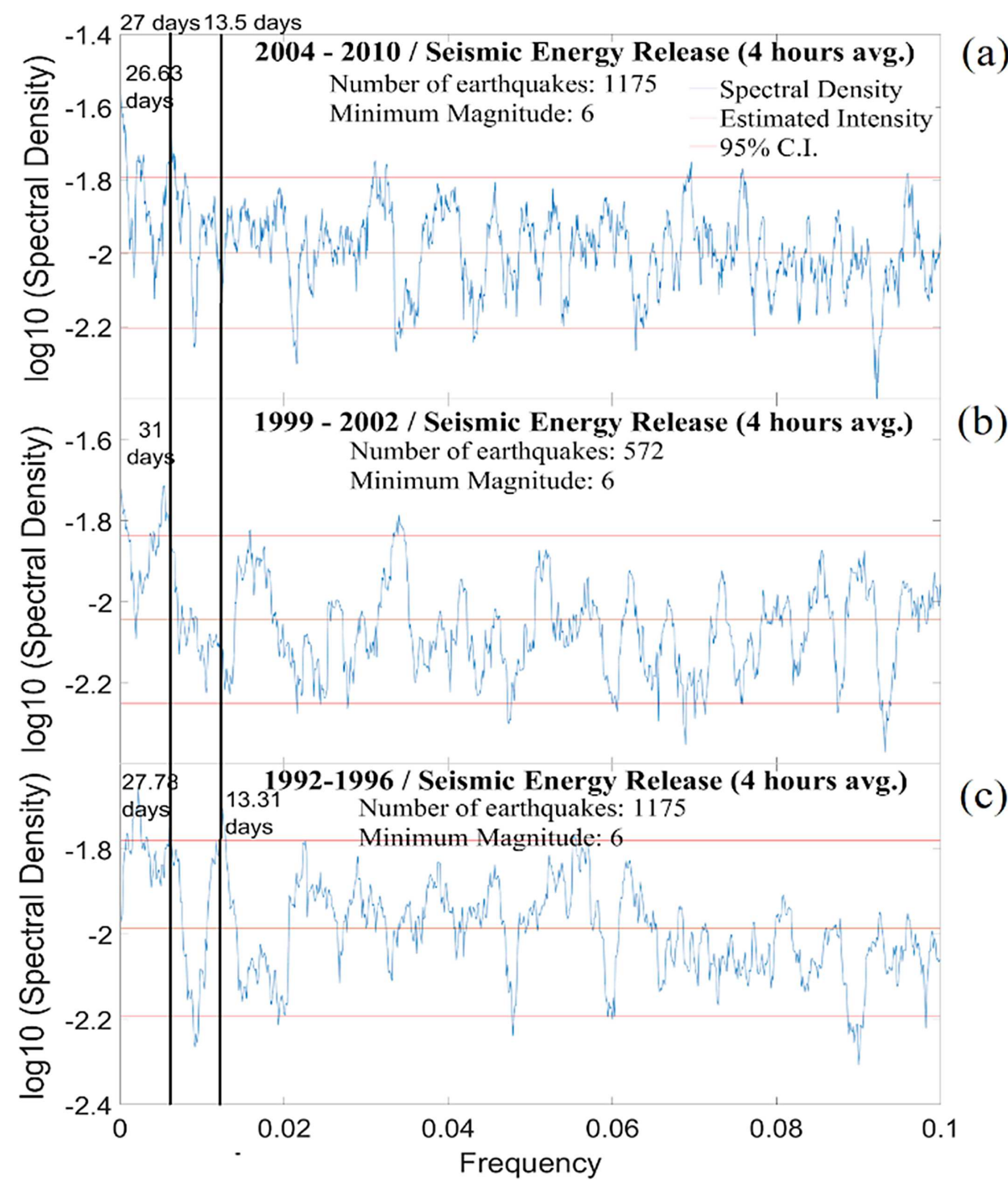

Fig. 13. Estimates of the logarithm to base 10 of the spectral density (blue lines) of the point process for seismic energy release (4 hours average) of EQs with magnitude $M \geq 6$ (a) during the decay phase and the deep minimum of SC23, between the years 2004-2010, (b) during the maximum phase of SC23 between the years 1999-2002, and (c) during the decay phase of SC22, in the time interval 1992-1996. The significant peak at 26.6 days during the transit from SC23 to $\mathrm{SC} 24$ (panel a) and the decay phase of $\mathrm{SC} 22$ (panel c) is very close to $\sim 27$ day mean solar rotation period (SRP). The significant peak at 31 days, during the solar maximum of SC23, is not much different to the SRP (see the detailed discussion in the text). Also, estimates of the logarithm to base 10 of the spectral density (blue lines) (d) of the time series for seismic energy release (1 day average) of EQs with magnitude $M \geq 1$ between the years 2004-2010 and (e) between the years 1999-2002. The significant peak at 26.6 (panel d) is very close to the solar rotation period of $\sim 27$ day (also in the case of EQs with magnitudes $M \geq 6$ during the transit from SC23 to SC24 in panel a), but the significant peak at 40.6 days (panel e), is considerably different from the mean solar rotation period. The red lines in Figure 3 indicate the 95\% confidence limits. They are the straight lines in panels a to c, because the power spectrum of the stationary point process tends to a constant as the frequency increases, whereas the power spectrum of the stationary process (time series) in panels d-e tends to zero as the frequency increases. 
normal geometry of the interplanetary magnetic field (IMF), and therefore that of the CH-driven HSS, for significant segments of time.

In order to check whether the surprising result of the 27-day periodicity is a common feature in seismological data and not a special finding during SC23, we extended our analysis to the solar cycle \#22. Figure 13c has been constructed as Figures3a-3b, but for $M \geq 6$ EQs which occurred during the decay phase of the previous solar cycle, SC22, between the years 1992-1996 (Fig. 3). From Figure 13c we also see that there is a clear peak at about the solar rotational period of $\sim 27$ days (27.78 days) and at the half 27-day period, which are significant at the $5 \%$ level. This result confirms that the 27-day periodicity in seismological data during the decay phase of the solar cycle is a characteristic feature of seismic activity for earthquakes $M \geq 6$. We point out that the clear peak at $\sim 13.31$ days of Figure 13c is due to the highly frequent presence of two CH sources of HSSs on the Sun during the decay phase of SC22 $[67,100]$. This result is also self-consistent with the concept of a strong relation between solar-seismological data.

Moreover, in order to check whether the solar influence concerns the EQs of low magnitudes as well, a similar procedure was applied as in Figures 13a-13c for a large amount of minor and moderate EQs including all of the EQs with $M \geq 1$. Here the data are daily and numerical since seismic $M \geq 1$ energy release can be estimated for each day. Figure 13d shows the estimates of the logarithm to base 10 of the spectral density (blue lines) of the energy release (one day average) from EQs with magnitude $M \geq 1$ between the years 2004-2010. Figure 13e shows again the same kind of data, but for the maximum phase of the solar cycle \#23, between the years 1999-2002, as was done previously for only large $(M \geq 6)$ EQs. The data shown in Figures $13 \mathrm{~d}-$ $13 \mathrm{e}$ have been obtained by smoothing the periodogram of the time series by using 17 adjacent ordinates and the red lines indicate approximate $95 \%$ confidence limits around a strongly smoothed spectral estimate [21].

In Figure 13d we can see that the significant peak which indicates a periodicity of 26.6 days at $5 \%$ level remains when all earthquakes with magnitudes $M \geq 1$ are considered as in the case of EQs with $M \geq 6$. Additionally, the same periodical modeling procedure is followed using, in this case, the autocorrelation function (Appendix A; Fig. 17), suggesting that a periodicity of $\sim 27$ days cannot again be rejected. This is an important new result. Can we actually attribute it to the contribution of smaller $(M<6)$ EQs or is it controlled by the presence of big earthquakes?

In Figure 13e we can see a significant peak at $5 \%$ level, which is present during the maximum phase of solar cycle \#23 (years 1999-2002), but at a period of $T_{M}=40.6$ days. We would like to discuss a little the much greater deviation of the peak of $M \geq 1$ earthquakes (40.6 days) compared to the peak of $M \geq 1$ EQs ( $T_{m}=26.6$ days) during the SC23 maximum phase from the mean solar rotation period ( $\sim 27$ days). The deviation of the spectral peak during the SC23 maximum phase $\left(T_{M}=40.6\right)$ is consistent with different physical conditions in the interplanetary space during the SC23 maximum from those at the following deep minimum, as we explained above, in the case of $M \geq 1$ EQs. Furthermore, we point out that since different solar conditions (minimum versus maximum) are related with different values of the spectral peaks (26.6/40.6) in the case of $M \geq 1$ EQs (panels $\mathrm{d}$ and e), while they are related with a spectral index of almost the same SRP in the case of $M \geq 6$ EQs (panels a-c), we might make the conclusion that the contribution of smaller $(M<6)$ EQs earthquakes make a real contribution to the results of the spectral analysis (if not, the peaks of Figs. 13c and 13e should coincide).

In conclusion, we found for the first time that seismological data during SC22 and SC23 show a periodicity at $\sim 27$ days and $\sim 13.5$ days during the decay phase of the solar cycle. This finding strongly supports the concept of the CH-driven HSSs (which corotate with the solar rotation period of $\sim 27$ days), as an important agent 
of the global terrestrial seismic activity. This means, of course, that the Sun strongly affects Earth's seismicity.

\section{Discussion}

\subsection{Solar and terrestrial seismic activity relationships: Conditions and limitations-A}

In a previous work, Anagnostopoulos and Papandreou [5] demonstrated that one month before the giant (M9.1) Sumatra-Andaman 2004 earthquake (EQ), six successive HSSs were all followed by strong to giant $(M>6.8)$ EQs. This catastrophic EQ occurred during the decay phase of SC23. Furthermore, a similar solar cycle dependence of seismic activity has already been noted in earlier studies. For instance, Simpson [110] examined solar and seismological data during the time period $1950-$ 1963 and came to the conclusion that the frequency of earthquakes appears to be highest during the period of the declining phases of both SC18 and SC19. Sobolev et al. [113] indicated a clear anticorrelation between global seismic energy output and the SSN.

On the same line, Kovalyov and Kovalyov [64] compared strong ( $M \geq 7.8)$ earthquakes with the solar cycle phases from 1900 up to 2004, and they found that strong earthquake occurrence is much less frequent when the sunspot number is high. Furthermore, Simpson [110] pointed out that other previous studies ([134]; [16]; [3]) studying much fewer earthquakes but over a much longer time span (as far back as the year 1750) appear to follow the same pattern. Odintsov et al. [81] performed the superposed epoch analysis method on solar and seismological data between the years 1900-1999 and they found a delay of the maximum seismic energy output compared to the year of solar maximum of three years, while Sytinskij [116] found a more complex pattern with three seismic energy maxima after the year of solar maximum for EQs with magnitudes $M \geq 7$. Shestopalov and Kharin [107] examined the time interval 1876-2012 and inferred that "the maximal seismic level of seismicity usually takes place during minimal solar activity and vice versa".

Based on the results presented in this paper, results from a variety of studies in order to further test the previous findings, i.e. that the seismic activity is in general higher during the period of the declining phase of the solar cycle, we investigate the possible mechanism of solar-terrestrial seismic triggering.

Concerning the solar cycle dependence, we confirmed previous results and we found that the global energy release of all EQs with magnitudes $M \geq 5.5$ between 1900-2017 shows in general higher values during the decay phase of the solar cycle, with a statistical significant peak three years after the solar maximum (Figs. 8 and 9). We also found that during the same period all of the 16 giant $(M \geq 8.5)$ EQs occurred during the decay, minimum and the rising phase of the solar cycle or during the maximum phase, but at times of a strong reduction of the SSNs, which are known to be related with an extension of the coronal hole areas and $\mathrm{CH}$-driven HSSs in the interplanetary space. Since the giant $(M \geq 8.5)$ EQs and the global energy release of all EQs with magnitudes $M \geq 5.5$ are related with CH-driven HSSs, we infer that the Sun is an important agent in triggering terrestrial seismic activity.

A very significant negative correlation $\left(r_{S}=-0.42, p<10^{-4}\right)$ was found between the trends of the SSN and the number of earthquakes with $M \geq 7$ during the period 1930-2010 [107]. Furthermore, we found a negative trend of association between the number of $M \geq 7.8 \mathrm{EQs}$ and the SSN during the last four solar cycles (SC22-SC24), with more EQs occurring in the decay phase of the solar cycles [97].

The negative correlation of the number of EQs with the SSN seems to have two limitations, one physical and another related with the method of the data analysis 
used. With regard to the first one, we found that the number of EQs -SSN anticorrelation is destroyed during long time periods of very low SSN amplitude solar cycles as at the beginning of the $20^{\text {th }}$ century $(1900-1930$; Figs. 8,10$)$, as in the cases of the Maunder (1645-1720) and the Dalton (1790-1820) minima. For a short time period when the number of EQs -SSN anticorrelation was destroyed in recent years we found that this was due to very low solar wind speed streams and low values of the IMF (2008-2009). The second reason for which one cannot see the number of EQs-SSN anticorrelation is artificial and it is due to the use of yearly averaged data. What can happen within a few months to transform the Sun's effects on the Earth's lithosphere?

The answer to this question is related with a major new result of this paper. We found that a sequence of CH-driven HSSs and CIRs, during a long time period ranging from some weeks to a few months, precedes the great EQs in the decay phase of the solar cycle, but also during the rising and the maximum phase, as confirmed by the twelve giant ( $M \geq 8.3$ ) earthquakes which occurred from 1980 to 2018, as well as in all EQs with $M \geq 8.5$ from 1900 until today. The CH-driven HSSs are most often observed during the decay phase, but also during the whole solar cycle. When a large coronal hole extends towards lower solar latitudes for a few months within the solar maximum phase, the monthly SSN drops, HSSs reach the Earth and then giant EQs can be observed. Therefore, a comparison of seismic activity with the annually averaged SSN can give misleading statistical results on the relationship between solar and terrestrial seismic activity.

A novel and very important result of the present study is the finding of a significant peak in the power spectrum of seismic energy release values during the decay phase of SC22 and during the transit phase of SC23-SC24 at 27 days, which is the rotation period of the Sun. This 27 -day periodicity should be attributed to the $\sim 27$ day quasi-periodic reappearance of $\mathrm{CH}$-associated $\mathrm{HSSs}$ and not to the influence of Earth's moon, since in the cases studied in detail we found a direct relation of several EQs with the CH-associated space HSSs (Fig. 3, Tab. 3 of the present paper, and the figures in the paper by Anagnostopoulos and Papandreou, [5]).

Most of our results can be checked in a simple way: (a) highest occurrence frequency of the giant $(M \geq 8.5)$ earthquakes and the global seismic $(M \geq 5.5)$ energy during the presence of $\mathrm{CH}$-driven HSSs, which are most often observed during the decay phase of the solar cycle and (b) the presence of solar rotation period of $\sim 27$ days in the global seismic energy during times of $\sim 27$-day repetitive HSSs.

\subsection{Solar and terrestrial seismic activity relationships. Conditions and limitations-B}

\subsubsection{The deep minimum of solar cycle 23 and the weak solar cycle 24}

A few giant earthquakes during SC24, including the catastrophic Japan 2011 EQ, occurred during unusual time periods compared to most giant EQs, which occurred during the decay phase: the rising (2010-2011) and the maximum phase (2012-2014) of SC24. The abundance of data gathered from many space missions during the recent solar cycles allows us to make solid conclusions, which can explain the "strange" features of the Sun-earthquake relationship.

The "strange" SC24 has been noted as such in the titles of several papers: "Is Something Wrong with the Present Solar Maximum?" [54]; "Why are there fewer large SEP events in the solar cycle?" [39]; "Why are Solar Energetic Particle Intensities so Much Lower in Solar Cycle 24, Especially at High Energies?" [78]. These special features of SC24 have been discussed from many points of view: solar activity $[14,17,27,79,126]$, interplanetary activity $[11,39,54,55,57,66,68,73,78,131,137]$. 
The results of these studies are consistent with our explanation (low solar wind speeds and low IMF magnitudes) about some deviations from the general negative correlation between SSN and seismic activity.

We saw that the deep minimum between SC23-SC24 with very low SSN is not related with high seismicity because of the low speeds of the solar wind streams at those times, whereas the "weak" SC24 allowed big EQs to occur because of enlarged coronal holes at those times (Figs. 3, 6, 8). The above two features of the solarterrestrial seismic relationship explains to some extent the reason for which the statistical results based on the comparison of SSN with the number of EQs $N_{\mathrm{E}}$ may sometimes not show a very strong and significant negative correlation. Any conclusion on the solar-terrestrial seismic relationship has to take into account the actual solar/interplanetary physical mechanisms, which affect the terrestrial seismicity.

\subsection{2 "The Solar-Terrestrial Triggering of Earthquakes is insignificant"?}

Love and Thomas ([69]; thereafter "LT") compared solar and terrestrial data and came to the conclusion that they cannot "reject the null hypothesis of no-solar terrestrial triggering of EQs" in the time interval 1900-2012, based on some cases and statistical studies. Here, we reexamine some of their hypotheses and conclusions in the light of the results of the present paper.

(1) LT noted that three historical and extremely large $(A A \geq 338.40 \mathrm{nT})$ CMErelated geomagnetic storms (Nov. 1941, $A A=340.74$, Nov 1941, $A A=340.74$; Québec, March 1989, $A A=338.41)$ were not followed by a significant increase in the number of EQs with $M \geq 7.5$ in the next month and the next year (LT; their Tab. 1). This fact has been explained as suggesting the absence of "any obvious relationship" between storms and seismicity. LT reached this conclusion by using, as a criterion, the arbitrary hypothesis that "the great storms generate great EQs". This hypothesis is not supported by the statistical studies we performed (Fig. 6). The big EQs are not in general related with CME-driven great storms, but, on the contrary, with CH-driven HSSs (Figs. 3-13).

(2) LT also claimed that the small SSN (related with calm geomagnetic conditions) in the month preceding the historical and catastrophic M9.5 Chile 1960 EQ, the M9.2 Alaska 1964 EQ and the recent M9.1 Sumatra-Andaman 2004 EQ (LT; Tab. 2) also suggests the absence of "any obvious relationship" between storms and seismicity. LT accepted as a criterion for their decision another arbitrary assumption, i.e. that the giant EQs are positively related with the level of the SSN. However, the 16 giant $(M \geq 8.5)$ EQs which we examined in this study (including the three EQs reported by LT), as well as the peaks in the seismic energy output during the years 1900-2017 were recorded during the decay phase of the solar cycle, or at times of reduced SSN during the maximum phase. Many statistical tests we performed imply a negative and not a positive correlation of the SSN with seismicity (with some limitations discussed above in Sects. 6.1 and 6.2).

(3) LT claimed that their statistical tests on solar and terrestrial data between 1900 and 2012 cannot reject the null hypothesis of no solar terrestrial triggering of EQs. Some more comments should be made here. The LT data analysis was based on the hidden hypothesis that the yearly value of the SSN shows a steady function with geomagnetic storms and an $M \geq 7.5 \mathrm{EQ}$ occurrence frequency. This is a very rough hypothesis for the complex character of the efficiency of solar activity in space processes, and it is easy to reject the null hypothesis of no solar terrestrial triggering of EQs. In this paper we have shown that the SSN is not independent of other parameters, as for instance, the long term variations of the SSN (Fig. 10), the 27-day periodicity of HSSs (Fig. 13), the changing efficiency of various solar cycle 
phases (Figs. 3, 6, 8), the variety of solar-magnetospheric effects (Fig. 6), etc. In addition, the yearly averages of solar, magnetospheric and seismic data used by LT in their statistical tests fail to adequately describe the solar influence on the terrestrial seismicity, since, for instance, giant EQs are often observed during large amplitude fluctuations of the monthly averaged SSN (Fig. 7).

(4) LT ignored the rich scientific literature, which provides evidence that higher seismic activity relates with the decay phase of the solar cycle, when 27 day-periodic $\mathrm{CH}-\mathrm{HSSs}$ interact with Earth and its environment. Therefore, instead of investigating the possible effect of the 27-day periodicity, they used monthly averaged data in order to avoid this space plasma modulation: "In general, solar-terrestrial conditions are often modulated with the 27 day rotation of the Sun, and, therefore, it is natural to consider average solar-terrestrial conditions over time scales of a month or so".

(5) From Figure 2a of LT we infer that during the years with high seismicity, as shown in the cases with more than 6 (5) EQs/yr with magnitudes $M \geq 7.5$, between 1963-2012, 29 (53) occurred with annual average sunspot number $G<50$, while only 14 (32) EQs occurred when the annual average index sunspot number was higher than 50. This anticorrelation between the annual values of the number of $M \geq 7.5$ EQs and the SSN is consistent with the results of Figures 3-5, and 7 of the present study. LT did not notice this anticorrelation during times of highest seismicity in their own data set, where they inferred that they cannot "reject the null hypothesis of no-solar terrestrial triggering of EQs".

It is obvious that LT case and statistical studies used the wrong hypotheses for a model of "solar-terrestrial triggering of earthquakes" and for this reason they inferred that this model does not work. However, their criticism provides a good contribution to the scientific communion to clarify open questions regarding the "solar-terrestrial triggering of earthquakes".

\subsubsection{Coronal hole-driven high speed streams, tectonic stress and ULF radiation before big earthquakes}

Here we discuss the possible compatibility of the CH-driven HSSs as an agent of large EQs with Pr. Freund's model (Fm) on the generation of several electromagnetic precursory phenomena of earthquakes. In order to explain pre-earthquake phenomena, Freund [33-35] noted that igneous and high-grade metamorphic rocks contain defects which, upon stressing, release defect electrons in the oxygen anion sublattice, known as positive holes. These charge carriers are assumed to be highly mobile, able to flow out of stressed rocks into surrounding unstressed rocks. The flow of positive holes form electric currents, which emit electromagnetic ULF radiation and generate other atmospheric electromagnetic phenomena, like mesospheric lightning, changes in the Total Electron Content (TEC), electric field turbulences etc. Here, it is crucial to point out that the term ULF radiation is used in general to cover a broad band of EM frequencies from $<1 \mathrm{mHz}$ to $<\sim 100 \mathrm{~Hz}$, that is a frequency range greater than five orders of magnitudes.

Several mechanisms proposed for the generation of ULF signals are based on the assumption that the primary process is the generation of some form of electrical potentials in the Earth' crust, which then lead to the generation of electric currents by pulling along charge carriers that are available in the rocks. These mechanisms hypothesize that potentials come first and electric currents come next. Pr. Freund' model is based on the recognition that mobile electronic charge carriers become activated in rocks when stresses are applied [34]. Under some conditions these charge carriers can flow through the Earth' crust, forming potentially powerful electric currents and generating EM radiation, specifically in the form of ULF signals. 
Freund and Pilorz ([30]; thereafter "FP") presented magnetic field measurements, obtained every 10 minutes, before the catastrophic M7.6 Chi-Chi Taiwan earthquake on September 21, 01:47:12 LT (17:47:12 UT), 1999. Measurements were analyzed for the period 1988-2001 and were compared with Fm. FP claimed that: (a) slow changes recorded between about 1996 and 1999 in the total magnetic field across Taiwan were caused by a telluric current flowing from East to West along the tectonic stress gradient, which is assumed to be the current of the stress-activated $h \cdot$ charge carriers and (b) ULF $(<1 \mathrm{mHz})$ waves preceding the Taiwan 1999 EQ could be a result of regional stress build-up processes mediated by stress-activated $\mathrm{h} \cdot$ charge flows.

A gradient in the number of EM ULF $(\sim 1-20 \mathrm{~Hz})$ events we found across the Hellenic (Greek) Arc, in south-west Greece and southward of the island of Crete ([8] Fig. 2 and 4), seems to be consistent with the concept of an electric current at the midline of boundary of tectonic plates frequently producing EQs; according to FP, if stress-activated positive hole currents flow along the stress gradient, and if they fluctuate, they should emit ULF. The long term trend of the regional magnetic field recorded at the Taiwan magnetometer station network shows a deviation from the IGRF model predictions starting some time in 1996/1997. This deviation may be related with a long term EQ preparation process.

Furthermore, our present study on solar/space weather influence on terrestrial seismicity along with the existing evidence of ULF $(\sim 1-20 \mathrm{~Hz})$ emissions related with seismic activity $[8,9,139]$ should be taken into account for some comparison of the FP interpretation on the magnetic field $<1 \mathrm{mHz}$ fluctuations observed just prior to the 1999 Chi-Chi M7.6 Taiwan EQ.

It is noticeable that the space weather during the time interval when the Chi-Chi M7.6 Taiwan EQ occurred has already been discussed in space science research. The time period August to September 1999 has been noted in the scientific literature as an unusual period with the most intense $(K p \geq 4+)$ geomagnetic activity that was caused by the arrival of a CH-driven HSS near Earth during the 20-years period, from 1993 until 2003 ([18,137], Tab. 1). It is anticipated that the CH-driven HSSs would produce $\sim 27 / 14$ day periodicity in a terrestrial environment and transfer Alfvén waves in the environment of Earth's magnetosphere. Indeed, sub-ULF ( $<1 \mathrm{mHz})$ wave activity was present at the ACE spacecraft in the near Earth interplanetary space, during the seven weeks before the Chi-Chi EQ and this wave activity was most evident between 12_14.9.1999(http://www.srl.caltech.edu/ACE/ASC/DATA/level3/mag/ magswesummary/ace_swe_mag_lv2_rtn_1999-252_1999-258.pdf; days 255-257, 1999), exactly during the period when pronounced ULF electromagnetic signals were recorded in Taiwan $[30,136]$. In agreement with a space agent of that ULF activity before the Chi-Chi EQ, 28.4-day and 14-day magnetic field periodicities were observed in Taiwan (FP); these periodicities are almost equal to the solar and the half solar rotation period, respectively.

On 12th of September 1999, the starting day of the strong ULF activity in Taiwan (FP), a sudden storm commencement (SSC) occurred, which was recorded by the terrestrial observatories (http://wdc.kugi.kyoto-u.ac.jp/kp/figs/kp1999.gif) followed by a great $\mathrm{CH}$-driven storm, with a geomagnetic activity index as high as $K p=6$.

The above relations between the solar-interplanetary space-magnetosphericlithospheric observations allow the assumption that the geomagnetic sub-ULF $(<1 \mathrm{mHz})$ magnetic field wave activity recorded in Taiwan before the Chi-Chi EQ may be of a space origin. The stressed rocks and the related positive hole current might be considered the agent of ULF emissions observed at higher $(>\sim 1 \mathrm{~Hz})$ frequencies [83].

The origin of ULF wave activity often observed before EQs has been disputed by several authors as EQ precursors. For instance, Campbell [22] compared magnetic 
field records from Fresno, Bolder and Tucson with magnetic field observations from Stanford University [36], near Loma Prieta, and he found that a similar trend for the $(<100 \mathrm{sec})$ ULF wave activity characterized all these locations. Campbell concluded that the ULF wave was not a local signal. He suggested that it was of a magnetospheric (solar) origin and he inferred that "An Earthquake Precursor Was Not Observed" in the case of the catastrophic M7.1 EQ in Loma Prieta, California.

We believe, based on the analysis of the previous sections, that the presence of the ULF wave activity before the Loma Prieta EQ was both an earthquake precursor and a solar-magnetospheric effect, and that this is probably the case for several other cases of pre-EQ $(<\sim 1 \mathrm{mHz})$ ULF wave events. However, this hypothesis needs statistical tests based on the comparison of Earth and space based observations. In other words, we need to check the hypothesis that, at least in some cases, the $(<\sim 1 \mathrm{mHz})$ ULF waves originate from space, and in this sense they are EQ precursory signals.

Finally, we infer that our comparison of the results of the present paper with the model of Pr. Freund as applied to the magnetic field observations before the Chi-Chi, Taiwan, 1999 EQ suggests that there is probably a limitation in the frequency band where Freund's model can apply. This hypothesis should be checked in future investigations based on the comparison of simultaneous space and terrestrial observations.

\subsection{Solar cycle dependence on electromagnetic earthquake precursors?}

Countless reports of precursory, mostly electromagnetic, phenomena have been accumulated from the analysis of data as recorded by ground and space based observations. These precursory signals are thought to reflect time-varying processes associated with the slow tectonic stress accumulation in the Earth's crust (Freund et al. 2017).

Some of these phenomena in Earth's crust and its environment concern (a) electromagnetic radiation at various frequency bands, as in ULF [8,9,139], VLF [80], TIR [120] and VHF bands [99], (b) plasma variations, as seen in observations of TEC and ionospheric plasma disturbances [4,49], radiation belt electron precipitation $[6,32,108],(\mathrm{c})$ chemical changes at or near ground [95] and local magnetic variations $[49]$.

Since the occurrence of great EQs is a solar cycle dependent phenomenon, the electromagnetic precursory events may present a dependence on the solar cycle as well. Here we discuss one of the EQ precursors, for which continuous observations are available for more than one solar cycle: the electron precipitation from the Van Allen (or radiation) belts. Radiation belt electron precipitation associated with broad band $(\sim 1-20 \mathrm{kHz})$ wave activity is very often observed before great EQs $[6,32]$.

Precipitation of relativistic electrons into the atmosphere has been suggested as an important loss mechanism for radiation belt electrons. EQ-related electromagnetic phenomena, Earth based VLF transmitters and lightning have been identified as agents of radiation belt electron precipitation $([6,32,108]$ and references therein). The "anomalous" sudden and short lasting $(\sim 2-3 \mathrm{~min})$ electron flux increases observed by satellites, due to electrons escaping from the Van Allen belts into the ionosphere have been called electron bursts (EBs). In order to examine any relation of these EBs with the solar cycle, in Figure 14 we compare the monthly mean of SSN (panel a) with the number of EBs, which are indicated by normal lines in panel b (adapted from Fig. 3 of a study by [32]) for the time period 1997-2016. The EBs presented in Figure 14b were observed by the NOAA-15 satellite [32]. An increase (decrease) in the length and the density of the normal lines suggests strong (weak) electron precipitation. 


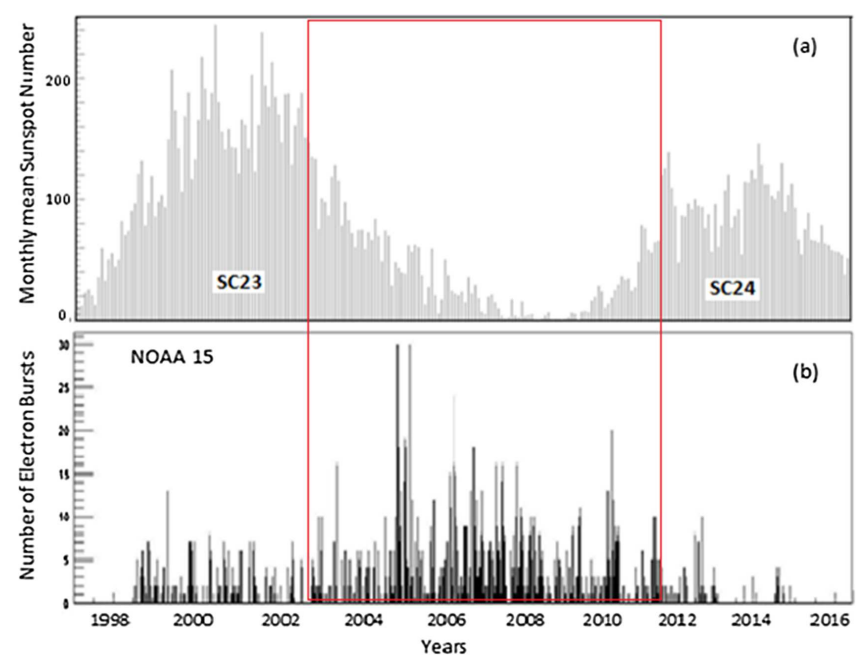

Fig. 14. Solar cycle variation of an earthquake electromagnetic precursor (radiation belt electron precipitation). In the figure, the monthly mean of SSN (panel a) is plotted along with the number of electron bursts (EBs) observed by the NOAA-15 satellite in the interval 1999-2002 (panel b) for the time period 1997-2016 (adapted from Fig. 3 of the paper by Fidani, 2018). High numbers of EBs are seen during the decay phase of SC23 and the rising phase of SC24. The EB temporal distribution between 1997 and 2016 follows the pattern of the HSSs and of the EQ frequency during SC23 and SC24.

A comparison between the data shown in the two panels demonstrates that the numerous strong EBs (long normal lines in high density) were observed mostly between the end of the year 2002 and the end of the year 2011 (interval indicated by a red rectangular), that is during an interval including the decay phase of SC23 and the rising phase of SC24, except for a period around the years 2008-2009, during the SC23-SC24 deep minimum. On the contrary, short normal lines in weak density are seen during the maximum phase of both SC23 and SC24. A comparison of these results with the results from Figures 3 and 6 suggests that the variations in the occurrence frequency of the EBs between the year examined (1998-2016) resembles that of great EQs.

Given that the solar cycle dependence of the EQ precursors is an issue not well investigated so far, we think that the above reported relation between EQ-related radiation belt electron precipitation events with the solar cycle (the decay phase of SC23 and the rising phase of SC24) is an important new finding. However, more work is needed to check this interesting relation.

Based on the existing evidence on ULF radiation during the preparation time of great EQs [9,139], we hypothesize that such a solar cycle dependence might be observed in ULF global radiation from the Earth to space. Such a work is in progress.

\subsection{A framework for the Physics of Space-driven earthquake triggering}

\subsubsection{The geomagnetic storms before enhanced Seismicity}

Since in this work we have provided significant evidence that the $\mathrm{CH}$-driven HSSs/CIRs are related with the terrestrial seismicity, the question is which physical mechanism is responsible for the external (solar/interplanetary space) energy transfer to the Earth's lithosphere The Earth's magnetosphere is comparatively large area 
extending up to $90000 \mathrm{~km}$ in the Sun-Earth direction and to very large distances in the nightside magnetosphere between the Earth's atmosphere and the interplanetary space Therefore if the CH-driven HSSs/CIRs is a major agent for provoking Earth's seismicity, the magnetosphere should mediate the HSSs/CIRs - lithosphere coupling

Many studies have provided significant evidence that there is a close relation between geomagnetic disturbances and seismic activity [12,81,112,138]. Urata et al. [128] analyzed the seismic data of a large number (4666) of earthquakes with $M \geq 6$ in relation to the variations of the $K p$ index from 1932 to 2016 and they confirmed a relation between geomagnetic field disturbances and earthquake occurrences. Moreover Anagnostopoulos and Papandreou [5] showed that a sequence of successive HSSs and enhanced geomagnetic activity preceded another series of EQs, by an average time of $\sim 1.5$ days, before the catastrophic M9.1 EQ in Sumatra-Andaman, December 26,2004 . The question, therefore, is what a disturbed magnetosphere can tell us about the solar- terrestrial seismic coupling. In order to elaborate this point, we should first examine the features of the two major types of storms, which are classified according to their two major triggering agents: the CMEs and the $\mathrm{CH}$-driven HSSs /CIRs.

Generally, the number of CME-associated storms is positively related with the solar cycle; both the CME rate at the Sun $[105,132,135]$ and the CME rate at $1 \mathrm{AU}$ $[100,102,103])$ increase during the solar maximum. CME-associated storms are large $\left(D_{\mathrm{st}}<\sim-100 \mathrm{nT}\right)$ and appear with an abrupt increase. Indeed, our analysis (Fig. 6) confirmed that the storms with a very abrupt initial phase $(\Delta K p \geq 3)$, are positively related with the solar cycle. In particular, we found that the yearly SSN and the yearly number of geomagnetic disturbances appearing with an abrupt initial phase show a strong correlation $r_{S}=0.96$, at a significance level $P<0.001$ (when the sets of normalized values were considered). These storms, with such an abrupt onset, have as triggering agent the CMEs [100].

The CH-HSS driven storms predominate for 3-4 years during the declining phase of the solar cycle and are the predominant drivers of weaker $(-75<D<-35 \mathrm{nT})$ storms with a slow initial phase $[11,100]$. It is remarkable that the general geoeffectiveness of $\mathrm{CH}$-driven HHSs is much greater than that of the CMEs; Badruddin et al. [11] identified 379 storms of the first type versus 62 of the second one, between 1996 and 2011.

In this paper we have found that higher seismicity is related with the $\mathrm{CH}$-driven HSSs, and, therefore, with weak storms, which show slow initial phase.

In a previous case study, we showed that a sequence of EQs was systematically preceded by another sequence of $\mathrm{CH} / \mathrm{HSS}$-driven storms before the giant (M9.1) and catastrophic 2004 Sumatra EQ [5]. In that study we found that out of 8 EQs with magnitudes $M \geq 6.2$ occuring within one month (November 25 -December 26, 2004), half of them were preceded by geomagnetic disturbances with an increase $\Delta K p=2$, and the other half with an increase $\Delta K p=3$ (Fig. 1b). None of the $\mathrm{CH} / \mathrm{HSS}$ storms preceded the 2004 Sumatra EQ showed values with $\Delta K p \geq 3$. As we mentioned, abrupt increases with $\Delta K p \geq 3$ is a feature of the CME-associated great storms.

Since enhanced seismicity is not correlated with the solar maximum (Figs. 3, 4a, 6-9) and great CME-associated storms with an abrupt (i.e. $\Delta K p \geq 3$ ) initial phase (Fig. 6), we may infer that the abrupt changes of the geomagnetic field is not the crucial physical mechanism in provoking EQ occurrence. This fact suggests that other physical parameters should play the major role in transferring energy from the Sun to the Earth's environment and provoking giant EQs or enhanced global seismicity; not the great storms with the abrupt increase.

However, the question is how we can explain the strong correlation between threehour Kp index and strong EQs during the month before the 2004 Sumatra-Andaman EQ. There are two ways to understand this finding under the special conditions 
before the giant Sumatra 2004 EQ: (i) under extreme space conditions (long time Alfvén wave activity), even a moderate $(2 \leq \Delta K p \leq 3) \Delta K p$ plays a secondary role to provoke great EQs, (ii) the $\Delta K p$ increase is not a parameter revealing the geomagnetic disturbance as a process related with the seismic activity, but it is a parameter reflecting other combined physical processes to the $\mathrm{CH}$-driven geomagnetic disturbances. Possible ways in which the CH-driven HSS/CIR may transfer energy to the lithosphere, through the magnetosphere, are discussed in the next final paragraph 6.4.2.

Finally, we would like to discuss the fact that in some cases a great EQ occurs about one day, or so, before or after a CME-associated storm. A representative example of an EQ occuring after a CME is the case of the catastrophic March 11, 2011 Japan M9.1 EQ. However, there are no significant statistical results supporting such a relation between CMEs-EQs. Furthermore, the CME reached the Earth's magnetosphere the day before the 2011 Japan EQ, in the presence of HSS activity starting as early as the end of January 2011 (data not shown here), that is at the end of a long time of electromagnetic energy transfer via Alfvén waves (see Sect. 6.4.2).

The opposite configuration, with (not a giant, but) a great EQ preceding a CME is the case of the Taiwan Chi-Chi 1999 EQ [30,95]. However, before the Taiwan 1999 EQ, a series of CH-HSSs associated with Alfvén wave activity also preceded the EQ. However, there is a possibility that the solar energetic particles $(1.91-4.75 \mathrm{MeV}$ protons), which arrived at Earth about a day before the Taiwan EQ and about two days before the geomagnetic storm (http://sd_www.jhuapl.edu/EPAM/data/ gif/lefs150_lems30_2268.gif), could have affected the ionospheric currents and contribute to the EQ triggering (Sarafopoulos; personal communication).

\subsubsection{The Physics of interaction of High Speed Streams with Earth's environment}

Although CMEs drive spectacular effects in the heliosphere and the Earth's magnetosphere, the results of our present study strongly suggest that $\mathrm{CH}$-driven HSSs seem to be the dominant space agent, which provokes EQs of all magnitudes on Earth (Figs. 3, 6-8 and 13). Besides the negative correlation found in general between SSN and seismic activity -in particular when monthly averaged data were used- the periodic variation of the global seismic activity with the solar rotation period of 27 days is a novel feature of seismological data. This periodicity poses severe problems to the models approaching seismicity as an autonomous terrestrial process separated from Earth's space environment and the Sun, which is the main energy source of the solar system. On the contrary, this finding strongly confirms the concept of CH-driven HSSs/CIRs, revealed from solar and space observations, as an important triggering agent of EQs.

From the 11 out of the 12 giant $(M \geq 8.3)$ EQs which occurred between 19802017 and shown in Table 3 (for which solar wind conditions could be examined from the ACE spacecraft that was launched in 1997), a sequence of HSSs associated with a quasi-permanent presence of Alfvén waves was found to precede the EQ occurrence for some weeks or a few months. Since the Alfvén wave activity is a special feature of the CH-driven HSSs, it is this process that mediates the CH-driven HSSs with terrestrial seismicity.

In general, since the incidence of HSSs and CIRs on the Earth's magnetosphere is well related with intense seismic activity, it is reasonable for seismology and earthquake prediction research to seriously take into account the space weather and also look for the candidate mechanism of space driven seismicity in the physics of CIR/ HSS and their interaction with Earth's electromagnetic environment (magnetosphereionosphere-lithosphere). 
Some first hypotheses can be made on the possible mechanism(s) of HSS EQ interaction based on the physics of HSSs/CIRs. As we mentioned above, the HSSs/CIRs causes weaker storms than the CMEs; the HSSs/CIRs-associated storms often present a $D_{\mathrm{st}}$ intensity between $-25 \mathrm{nT}$ and $-75 \mathrm{nT}$ and typically do not reach intensities of $D_{\text {st }}<-100 \mathrm{nT}$. However, whereas CME-driven storms usually last a few hours, the recovery phases of CIR-induced magnetic storms can last for a few days, up to 27 days, [123]. Furthermore, since during a quiet Sun HSSs/CIRs corotate with the Sun's 27-day period, one or more (most often two) CH-driven HSSs, can almost continuously affect the Earth's magnetosphere even for some months. During such long time periods HSSs/CIRs can transfer much more energy in the magnetosphere than a single CME. This is probably the reason of the relation found between seismicity and CH-driven HSSs.

Large amplitude Alfvén waves are embedded in HSSs (Fig. 11). This is a special feature of CH-associated HSSs compared to the CME-associated HSSs, as we mentioned above, and, therefore, it is reasonable to hypothesize that these waves might be an actual agent of the space - lithosphere electromagnetic coupling, and a counterpart of the lithospheric stresses in provoking terrestrial seismicity. Two scenarios could be considered the most serious candidates for the Alfvén wave activity to transferring electromagnetic energy into the lithosphere: (a) either through magnetic reconnections or /and (b) a long time siege of lithosphere by ULF waves.

(a) Geomagnetic storms via magnetic reconnections transfer energy from the solar wind into the magnetosphere. The southward component of the interplanetary magnetic field (IMF) interacts with Earth's northward magnetic field and triggers magnetic storms. During times of $\mathrm{CH}$-associated HSSs, energy transfer can be in progress during semi-continuous shallow plasma injections, due to quasi-periodic magnetic reconnections of the southward component of the Alfvén waves with magnetopause fields. Therefore, the prolonged presence of Alfvén waves during a sequence of HSSs/CIRs may lead to almost continuous driving of the magnetosphere [57] for a long time period of a few to several weeks and energy transfer to the lithosphere.

The next step to examine is which way the magnetosphere mediates the energy transfer to the ionosphere. Can the almost continuous, but weak geomagnetic storms stimulate significant effects on the ionosphere? Tsurutani and Gongalez [125] suggested that during times of continuous presence of Alfvén waves, almost continuous UV Auroras often cover the entire dayside and nightside auroral zones, which can last for days to many weeks. This phenomenon is called HILDCAAs (High Intensity Long Duration Continuous AE Activity). During such periods, disturbances in ionospheric electric fields and thermospheric winds caused by the plasma injection at high latitudes affect the middle and the low latitude ionosphere [2]. It is remarkable that Abdu et al. notes that this phenomenon is more efficient during corotating $\mathrm{CH}$-associated HSSs. Once ionospheric currents enhance, they can interact with the lithosphere.

The varying ionospheric currents induce currents in the Earth's lithosphere. Then magnetic moment of the induced currents may interact with the horizontal intensity $\mathrm{H}$ of the main Earth's magnetic field, and a torque results from this process acting on a current sheet on the lithosphere. Duma and Ruzhin [29] claimed that Sq currents in the ionosphere can play this role.

On the other hand, Odintsov et al. [81], based on the work of Prikryl et al. [93], suggested that as the auroral electrojet strengthens, during storms, and generates atmospheric gravity waves, which are transmitted downwards. Then the westward zonal winds strengthen and the surface air pressure changes. The pressure balance on tectonic plates is disrupted and if enough tension is accumulated, an earthquake is triggered. 
(b) It is well known that ULF waves are often observed at Earth-based observatories $[62,76]$. It is also known that changes in orientation of the interplanetary magnetic field or an increase in solar wind speed can have dramatic effects on the type of waves seen on the Earth. Solar wind produces a wide variety of ULF wave types that are classified on the ground as either Pi or Pc pulsations. The location of the projections of these regions onto the Earth depends on the solar wind dynamic pressure and the magnetic field. The properties also depend on the conductivity of the Earth underneath the observer [71].

The solar wind is a source of ULF waves observed at Earth, particularly at low frequencies around $1 \mathrm{mHz}$ [13]. Some of these waves originate at the Sun and are both carried by, and propagate through, the solar wind. Besides solar wind, waves produced upstream from the Earth's bow shock, bow shock waves, and the magnetopause are sources of ULF waves penetrating into the magnetosphere. The magnetopause is a source of several types of ULF waves. One type is produced by oscillatory dynamic pressure fluctuations in the solar wind [58]. The corresponding ULF signal seen at the ground is in general an electromagnetic wave radiating from currents induced in the ionosphere, and not the magnetohydromagnetic waves themselves.

Sarafopoulos [106] has demonstrated that sometimes monochromatic or quasiperiodic geomagnetic pulsations have been exo-magnetospherically excited by a wavesource with a periodicity from $\sim 3$ to $\sim 10 \mathrm{~min}$ and that the magnetosphere in these cases has the ability to respond to solar wind ULF waves in such small time scales as $2.7 \mathrm{~min}$.

Most interestingly, many observations obtained simultaneously in space and on Earth have shown that magnetospheric perturbations produced by an external source deeply penetrate into the magnetosphere and that the lower-latitude data on the nightside are important in monitoring the external source variations [61,130]. Moreover, Russell et al. [98] reported that the best correlation between ground level changes and the change in the solar wind dynamic pressure occurs at geomagnetic latitudes from $15^{\circ}$ to $30^{\circ}$.

Campbell [22] compared magnetic field records from Fresno, Bolder and Tucson with magnetic field records from Stanford University [36], near Loma Prieta, and he inferred that the ULF waves observed before the catastrophic M7.1 EQ in Loma Prieta, California, in October 1989, was of a space origin. The 1993 Guam M7.8 EQ [48] is also mentioned as a characteristic case in which ULF wave activity appears to be an EQ precursor [48]. A comparison of the ULF wave activity with magnetic field and plasma observations made by the IMP-8 satellite (data not shown here) suggest that the August 8, 1993 Guam EQ occurred within a CIR lasting from 4 to 11 August, in the presence of strong Alfvén wave activity. We point out that these observations were obtained during the decay phase of SC22 (Fig. 3b; 2nd EQ after SC22 maximum phase).

In conclusion, a comparison of observations of Earth and space based observations before some EQs suggests that the ULF $(<1 \mathrm{mHz})$ are well correlated with CH-HSS related Alfvén waves at the ACE spacecraft, outside the Earth's magnetosphere (data not shown here). These results will be presented in a future paper. Furthermore, a statistical study will be needed to check, to what extent, the Alfvén waves may be correlated with the $(<1 \mathrm{mHz})$ ULF waves observed before great EQs. In this case the $(<1 \mathrm{mHz})$ ULF would be not only an EQ precursor but also an agent of the seismic events. 


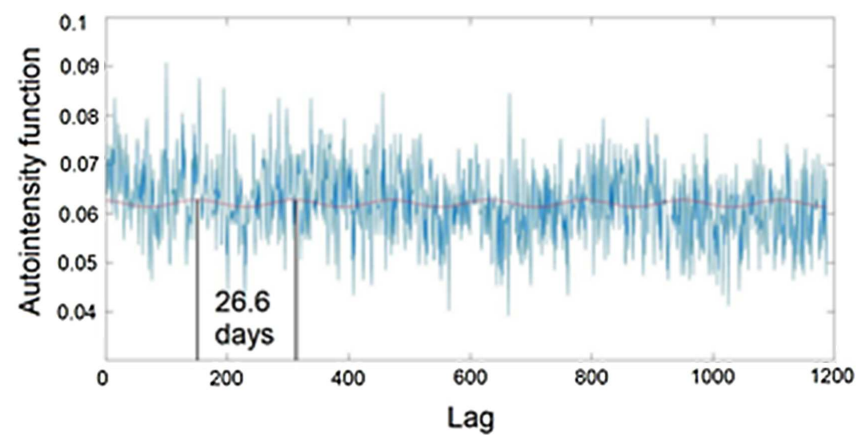

Fig. 15. Estimate of the autointensity function (blue line) of the point process for the seismic energy release (4 hours average) of EQs with magnitude $M \geq 6$ between the years 2004-2010 and the model of the form (red line).

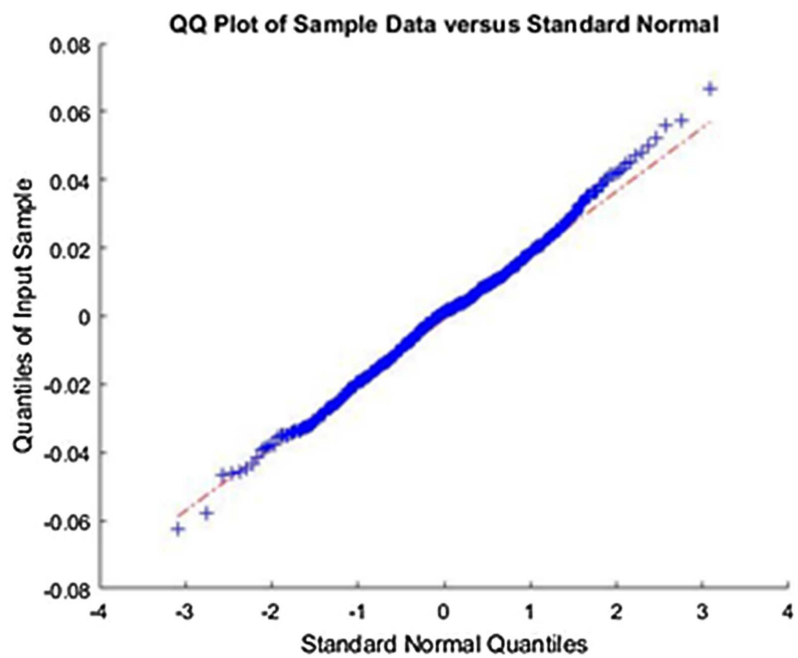

Fig. 16. Q-Q plot of the residuals between the estimated autointensity function of the point process for seismic energy release ( 4 hours average) of EQs with magnitude $M \geq 6$ in the time interval 2004-2010 and the model of the form.

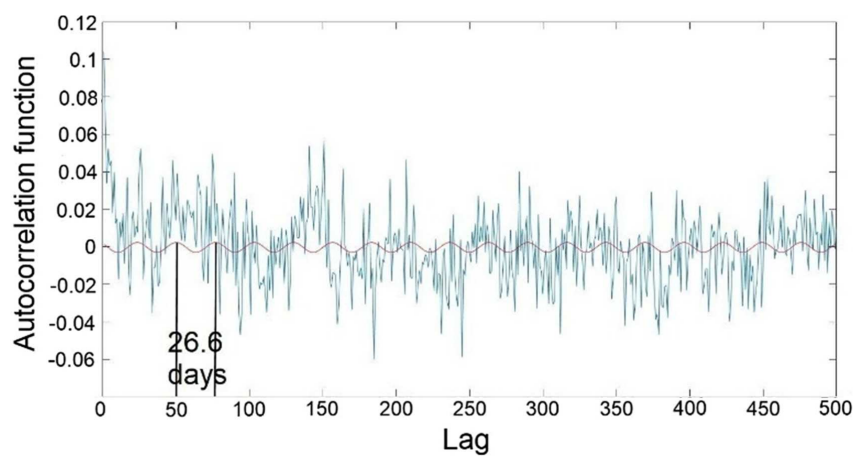

Fig. 17. Estimate of the autocorrelation function (blue line) of the time series for seismic energy release of EQs with magnitude $M \geq 1$ in the time interval 2004-2010 and the model of the form $a 0+a 1 \operatorname{sint}+a 2 \operatorname{cost}$ (red line). 


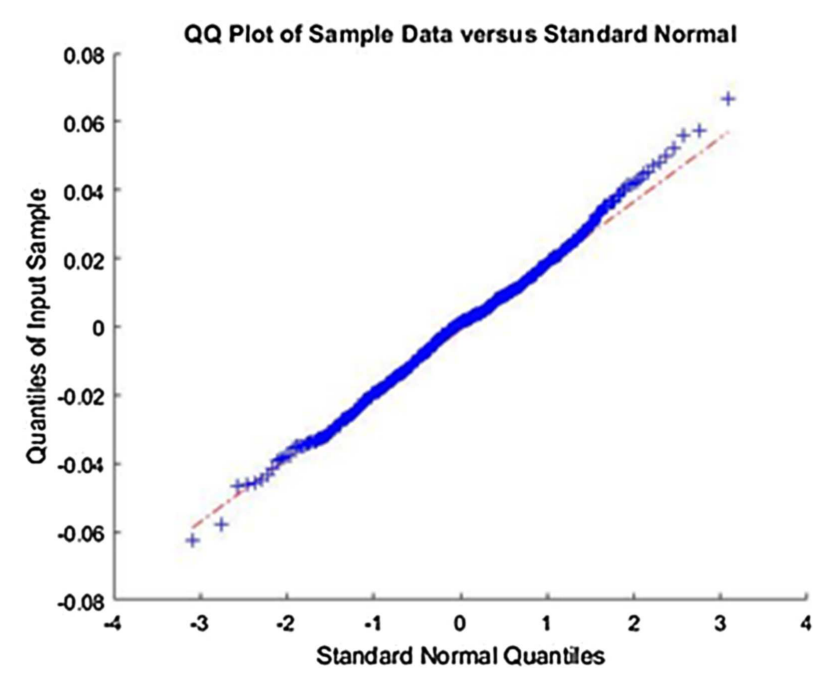

Fig. 18. Q-Q plot of the residuals between the estimated autocorrelation function of time series for seismic energy release of EQs with magnitude $M \geq 1$ in the time interval 2004-2010 and the model of the form $a 0+a 1$ sint $+a 2 \operatorname{cost}$.

\section{Conclusions}

We believe that, although, a precise mechanism of the HSSs - EQs electromagnetic interaction needs much more work to be completely understood, the concept of the corotating high speed solar wind streams as a dominant triggering agent of EQs opens a new insight into the complex ways of earthquake appearance. This study contributes to the general concept supported by many other previously published papers that our Earth cannot be considered an autonomous system in our solar system, separated from its electromagnetic environment. It also reminds us that Earth is a planet of our close star: the Sun. We believe that there is significant observational evidence suggesting that the Sun, interplanetary space, the magnetosphere, the ionosphere, the Earth's atmosphere, and the lithosphere reveals an entire physical system, where seismicity should be considered as a part of integrated physical processes in our solar system.

\section{Glossary and Acronyms.}

Alfvén waves. A low-frequency (compared to the ion cyclotron frequency) travelling oscillation of the ions and the magnetic field in a plasma, in the same direction and transverse to the direction of propagation. The Alfvén waves are compressed waves propagating in the direction of the magnetic field.

AU: Astronomical Unit. It is a unit of length, roughly the distance from Earth to the Sun. $1 \mathrm{AU} \approx 150$ million $\mathrm{kms}$

$(\mathrm{CH})$ : Coronal Holes. Coronal holes are the darkest patches on the solar surface, as measured in ultraviolet (UV) and X-ray radiation and least active regions of the Sun. Coronal holes are regions of low density plasma (low temperature) on the Sun that have magnetic fields opening freely into the heliosphere and they are associated with rapidly expanding open magnetic fields and the acceleration of high-speed solar wind. The term "coronal hole" has come to denote several phenomena that may not always refer to the same regions. A more theoretical usage equates coronal holes with all open-field footprints of time-steady solar wind flows. 
(CIR): Corotating Interaction Region. The flow of plasma from the solar corona is non-uniform in both time and space. The fast wind catches up with upstream slow wind and a compressive region is formed at the interface of the two streams. These structures reappear with the $\sim 27$ days rotation period of the Sun. When these coronal hole-associated streams are long lasting, they lead to the formation of corotating interaction regions.

(CME): Coronal Mass Ejections. Large expulsions of plasma and magnetic field from the Sun's corona and released into the solar wind. They can eject billions of tons of coronal material. CMEs most often originate from active regions on the Sun's surface, such as groupings of sunspots, and they are often related with solar flares. CMEs most often occur during solar maxima.

(EM): Electromagnetic.

Flare: A rapid release of energy in the solar atmosphere which has the result of temperature increase and the acceleration of relativistic electrons (sometimes acceleration of protons too). From this event, particles, waves, radiations propagate in the interplanetary space and arrive on Earth environment.

Geomagnetic storms: A geomagnetic storm is a major disturbance of Earth's magnetosphere caused by different sources on the Sun. The increase in the solar wind pressure initially compresses the magnetosphere. The solar wind's magnetic field interacts with the Earth's magnetic field and transfers an increased energy into the magnetosphere.

(HSS): High Speed Streams. "Fast" solar wind emanating from coronal holes on the Sun and observed at Earth with speeds as high as $800 \mathrm{~km} / \mathrm{s}$.

(IMF) Interplanetary Magnetic Field

Magnetopause: The boundary between the Earth's magnetic field and the solar wind.

(MHD): Magnetohydrodynamics. Hannes Alfvén combined the mathematics of fluid mechanics and electromagnetism to predict that plasmas could support wavelike variation in the magnetic field. MHD is the branch of physics that studies the behavior of an electrically conducting fluid, such as plasma, in magnetic fields (the study of magnetized plasma). Alfvén received the Nobel prize in 1970.

Plasma: One of the four fundamental states of matter. It is a quasineutral gas of charged particles which exhibits collective behavior. The Earth's ionosphere is a plasma and the Earth's magnetosphere contains plasma. The Sun is an example of fully ionized plasma, along with the solar corona and stars.

Solar wind: A stream of charged particles, primarily electrons and protons, flowing outward from the upper atmosphere of the Sun. Embedded within the solarwind plasma is the interplanetary magnetic field. Solar wind is a plasma emanating from the Sun into the interplanetary space.

(SSC) (Storm): Sudden Commencements. An abrupt increase or decrease in the northward component of the geomagnetic field, which marks the beginning of a geomagnetic storm or an increase in activity lasting at least one hour. The sudden commencements are defined from magnetograms of the worldwide network of magnetic observatories.

X Flare: The classification system for solar flares uses the letters A, B, C, M or $\mathrm{X}$, according to the peak flux in watts per square meter $\left(\mathrm{W} / \mathrm{m}^{2}\right)$, as measured at the Earth by the GOES spacecraft. X Flare is a very intense flare $\left(>10^{-4} \mathrm{~W} / \mathrm{m}^{2}\right)$.

The leading author thanks Pr. K. Tsinganos and Pr. I Daglis for helpful discussions on significant issues of the present study during his sabbatical in the University of Athens and Ass. Pr. P. Preka-Papadema for her kind invitation. He also thanks the Academician Dr. S.M. Krimigis and Dr. Patsis for their comments and discussion during his short visit in the Research Center for Astronomy and Applied Mathematics of the Academy of Athens 
(November 2017). He also thanks Pr. F. Freund and G. Ouillon for their kind invitation to contribute to the present special journal issue. The authors thank the PIs and the teams/Institutions providing the online data of the EPAM- MAG-SWEPAM onboard ACE satellite and of the Atmospheric Imaging Assembly (AIA) onboard the Solar Dynamics Observatory (SDO) satellite as well as World Data Center for Geomagnetism, Kyoto.

Open Access This is an open access article distributed under the terms of the Creative Commons Attribution License (https://creativecommons.org/licenses/by/4.0/), which permits unrestricted use, distribution, and reproduction in any medium, provided the original work is properly cited.

Publisher's Note The EPJ Publishers remain neutral with regard to jurisdictional claims in published maps and institutional affiliations.

\section{Appendix A: Autointensity function}

The autointensity function can be considered as the conditional probability density of an event of a stationary point process occurring at time $t+h$ given that an event occurred at time $t$ for various values of $h$. The autointensity function shows the relation that exists between the points of a stationary point process in contrast to the autocorrelation function that shows the relation that exists between any two values of the time series. In Figure 15, we present estimates of the autointensity function (blue line) of the point process for seismic energy release (four hours average) of earthquakes with magnitude $M \geq 6$ between the years 2004-2010. It appears that in the autointensity function there is a periodicity which we approached with a model of the form

$$
a_{0}+a_{1} \sin \omega t+a_{2} \cos \omega t
$$

(red line) and we proved that it is a good approach for the periodicity by using the Q-Q plot (see Fig. 16). A Q-Q (quantile-quantile) plot is a probability plot that is to say a graphical method for comparing two probability distributions by plotting their quantiles against each other [37]. In Figure 16 we compare the probability distribution of the residuals of the model of the form (A.1) with the standard normal distribution.

A fitted model of the form (A.1) for period of $\sim 27$ days seems to be a good fit as the residuals do not show serious deviations from normality as described in Figure 16 [19]. Also, the powerful Anderson - Darling test [96] gives a p-value of 0.1 indicating that the null hypothesis that the residuals follow a standard normal or the autointensity function presents a periodicity of $\sim 27$ days cannot be rejected.

\section{Appendix B: Autocorrelation function}

If we use a model of the form (A.1) (Fig. 17), then the null hypothesis that the autocorrelation function presents a periodicity of $\sim 27$ days in the time series for energy release (1 day average) of EQs with magnitude $M \geq 1$ in the time interval 2004-2010 cannot be rejected. This follows again by the Anderson - Darling test $(\mathrm{p}$-value $=0.1162)$ and the $\mathrm{Q}-\mathrm{Q}$ plot of the residuals (Fig. 18) which shows that there are no serious deviations from normality.

\section{References}

1. H. Alfvén, Cosmical electrodynamics (Clarendon Press, Oxford, 1950)

2. A.A. Abdu, J.R. de Souza, J.H.A. Sobral, et al., Magnetic Storms Associated Disturbance Dynamo Effects, in The Low and Equatorial latitude Ionosphere, Magnetic 
Storms Caused by Corotating Streams, Geophysical Research Series 167, Recurrent Magnetic Streams, AGU, 283 (Wiley, 2006)

3. V.I. Afanas'yeva, Geomagn. Aeron 3, 561 (1963)

4. M. Akhoondzadeh, M. Parrot, M.R. Saradjian, Nat. Hazards Earth Syst. Sci. 10, 7 (2010)

5. G.C. Anagnostopoulos, A. Papandreou, Nat. Hazards Earth Syst. Sci. 12, 1551 (2012)

6. G.C. Anagnostopoulos, E. Vassiliadis, S. Pulinets, Ann. Geophys. 55, 21 (2012)

7. G.C. Anagnostopoulos, S.A. Menesidou, V.G. Vassiliadis, A. Rigas, Correlation between Solar Energetic Particle events and Earth's surface Temperature in NorthEast USA 12th HelAS_Conference (Thessaloniki, 2015), https://www.researchgate. net/publication/309490064_Correlation_between_Solar_Energetic_Particle_ events_and_Earth's_surface_Temperature_in_North-East_USA

8. M.A. Athanasiou, G.C. Anagnostopoulos, C.N. David, et al., Res Geophys. 4, 5001 (2014)

9. M.A. Athanasiou, G.C. Anagnostopoulos, A.C. Iliopoulos, et al., Nat. Hazards Earth Syst. Sci. 11, 1091 (2011)

10. B. Badruddin, A. Kumar, Solar Phys. 290, 127 (2015)

11. B. Badruddin, F. Mustajab, M. Derouich, Adv. Space Res. 62, 765 (2018)

12. V. Bakhmutov, F. Sedova, T. Mozgovaya, Publ. Inst. Geophys. Pol. Acad. Sci. 43, 931 (2007)

13. A. Barnes, in Solar-Terrestrial Physics: Principles and Theoretical Foundations, edited by R.L. Carovillano, and J.M. Forbes (D. Reidel Publishing Company, Dordrecht, 1983), pp. $155-199$

14. S. Basu, J. Phys.: Conf. Ser. 440, 012001 (2013)

15. J. Vencloviene, R. Babarskiene, R. Slapikas, Nat. Hazards 65, 1 (2013)

16. P. Bernard, Compt. Rend. 250, 2738 (1960) (in French)

17. D. Biesecker, NOAA/Space Environ. Center 1-10, 2011

18. J.E. Borovsky, M.H. Denton, J. Geophys. Res. 111, A07S08 (2006)

19. G.E. Box, G.M. Jenkins, G.C. Reinsel, G.M. Ljung, Time Series Analysis: Forecasting and Control (John Wiley and Sons, New York, 2015)

20. D.R. Brillinger, J. R. Stat. Soc. B 38, 60 (1976)

21. D.R. Brillinger, Time Series: Data Analysis and Applications (Holt Rinehart and Winston Inc., New York, 1981)

22. W.H. Campbell, J. Geophys. Res. 114, A05307 (2009)

23. C. Chatfield, The Analysis of Time Series: An Introduction, 5th edn. (Chapman \& Hall London, 1996)

24. M.A. Clilverd, C.J. Rodger, R.M. Millan, et al., J. Geophys. Res. 112, A12206 (2007)

25. R.S. Cranmer, Liv. Rev. Sol. Phys. 6, 3 (2009)

26. M. Denton et al., EOS Trans., AGU, 89, 62 (2008)

27. M. Dikpati, P.A. Gilman, G. Toma, R. Ulrich, Geophys. Res. Lett. 37, 14 (2010)

28. L. Doda, Air Fleet. 3, 56 (2001)

29. G. Duma, Y. Ruzhin, Nat. Hazards Earth Syst. Sci. 3, 171 (2003)

30. F. Freund, S. Pilorz, in The Frontier of EQ Prediction Studies, edited by M. Hayakawa (2012), pp. 468-508

31. F.T. Freund, G. Ouillon, J. Scoville, D. Sornette, Eur. Phys. J. Special Topics 230, 7 (2021)

32. C. Fidani, Terr. Atmos. Ocean Sci. 29, 117 (2018)

33. F.T. Freund, Acta Geophys. 58, 719 (2010)

34. F.T. Freund, J. Asian Earth Sci. 41, 383 (2011)

35. F.T. Freund, Acta Geophys. 61, 775 (2013)

36. A.C. Fraser-Smith et al., Geophys. Res. Lett. 17, 1465 (1990)

37. A. Ghasemi, S. Zahediasl, Int. J. Endocrinol. Metab. 10, 486 (2012)

38. M. Gerontidou, H. Mavromichalaki, T. Daglis, Sol. Phys. 293, 131 (2018)

39. J. Giacalone, R.A. Mewaldt, Why are there fewer large SEP events this solar cycle? American Geophysical Union Fall General Assembly Abstract idSH32A-05 (2016)

40. J.T. Gosling, D.J. McComas, J.L. Phillips, et al., J. Geophys. Res. 96, 7831 (1991) 
41. M.N. Gousheva, K.Y. Georgiva, B.B. Kirov, et al., On the relation between solar activity and seismicity, in Proceedings of the International Conference on Recent Advances in Space Technologies RAST '03 Istanbul Turkey (2003), pp. 236-240

42. R.S. Granmer, Living Rev. Solar Phys. 6, 3 (2009)

43. L.J. Gray, J. Beer, M. Geller et al., Rev. Geophys. 48, 1 (2010)

44. P.J. Green, B.W. Silverman, Nonparametric Regression and Generalized Linear Models: A Roughness Penalty Approach (Chapman and Hall London 1994)

45. T. Gulyaeva, Development Earth Sci. 2, 14 (2014)

46. B. Gutenberg, C.F. Richter, Ann. Geofis. 9, 1 (1956)

47. M. Hayakawa, Sensors 7, 1141 (2007)

48. M. Hayakawa, R. Kawate, O.A. Molchanov, et al., Geophys. Res. Lett. 23, 241 (1996)

49. M. Hayakawa, K. Hattori, K. Ohta, Sensors 7, 1108 (2007)

50. D.J. Herdiwijaya, J. Arif, M.Z. Nurzaman, AIP Conf. Proc. 1677, 050003 (2015)

51. J.R. Herman, R.A. Goldberg, Sun Weather and Climate (Dover Pub Inc., New York, 1978)

52. Y.-Y. Ho, J.-Y. Liu, M. Parrot et al., Nat. Hazards Earth Syst. Sci. 13, 3281 (2013)

53. J. Jakubcova, M. Pick, Ann. Geophys. 5, 135 (1987)

54. Y. Kamide, K. Kusano, Space Weather 11, 140 (2013)

55. Y. Kamide, K. Kusano, Space Weather 13, 365 (2015)

56. R.P. Kane, Adv. Space Res. 39, 189 (2007)

57. A. Kavanagh, M. Denton, Astron. \& Geophys. 48, 6.24 (2007)

58. L. Kepko, H.E. Spence, H. Singer, Geophys. Res. Lett. 29, 39.1 (2002)

59. V.E. Khain, E.N. Kalilov, Trans. Inter. Acad. Sci. 3, 217 (2008)

60. E.K.J. Kilpua, A. Balogh, R. von Steiger, et al., Space Sci. Rev. 212, 1159 (2017)

61. K.-H. Kim, C.A. Cattell, D.-H. Lee et al., J. Geophys. Res. 107, 1406 (2002)

62. G.M. Kivelson, C.T. Russell, Introduction to Space Physics (Cambridge University Press, Cambridge, 1995)

63. V.V. Kormiltsev, N.P. Kostrov, A.N. Ratushnyak et al., The influence of electroosmotic pressure generated by geomagnetic disturbances on the evolution of seismotectonic process, in Electromagnetic Lithosphere-Atmosphere-Ionosphere Coupling, edited by M. Nayakawa, O.A. Molchanov (Terrapub, Tokyo, 2002), pp. 203-207

64. M. Kovalyov, S. Kovalyov, arXiv: 1403.5728v2 [physics.gen-ph] (2015)

65. K. Kudela, M. Storini, M.Y. Hofer et al., Space Sci. Rev. 93, 153 (2000)

66. S.E. Kozyra et al., Magnetic Storms Caused by Corotating Streams Geophysical Research Series, Recurrent Magnetic Streams, AGU, 167 (2006)

67. D. Lario, R.B. Decker, S. Livi et al., J. Geophys. Res. 110, A09S11 (2005)

68. D. Lario, R.B. Decker, E.C. Roelof, et al., J. Phys.: Conf. Ser. 900, 012012 (2017)

69. J.J. Love, J.N. Thomas, Geophys. Res. Lett. 40, 1165 (2013)

70. C. Lowder, J. Qiu, R. Leamon, Solar Phys. 292, 18 (2017)

71. R.L. McPherron, Magnetotails in the Solar System (2015)

72. C.J. Qiu, R. Leamon, Sol. Phys. 292, 18 (2017)

73. J.G. Luhmann, C.O. Lee, Y. Li et al., Sol. Phys. 256, 285 (2009)

74. L.N. Makarova, A.V. Shirochkov, On the connection between the Earth's magnetosphere magnetopause position and the earthquakes occurrence, Abstracts of XXVI General Assembly LJRSI (Toronto, Canada, 1999)

75. H. Mavromichalaki, A. Vassilaki, E. Marmatsouri, Sol. Phys. 115, 345 (1988)

76. R.L. Mcpherron, Surv. Geophys. 26, 545 (2005)

77. A.A. Melkumyna, A.V. Belov, M.A. Abunina et al., Geomagn. Aeron. 58, 154 (2018)

78. R.A. Mewaldt, G. Li, C. Cohen et al., Why are Solar Energetic Particle Intensities so Much Lower in Solar Cycle 24 Especially at High Energies? American Geophysical Union Fall General Assembly abstract idSH41D-01 (2016)

79. D. Nandy et al., Nature 471, 80 (2011)

80. F. Nemec, O. Santolık, M. Parrot, J. Geophys. Res. 114, 72 (2009)

81. S. Odintsov et al., Phys. Chem. Earth 31, 88 (2006)

82. S.D. Odintsov, G.S. Ivanov-Klodonyi, K. Gergieva, Bull. Russ. Acad. Sci. Phys. 71, $593(2007)$ 
83. K. Ohta, K. Umeda, N. Watanabe, et al., Nat. Hazards Earth Syst. Sci. 1, 37 (2001)

84. S.J. Palmer, M.J. Rycroft, M. Cermack, Surveys Geophys. 27, 557 (2006)

85. S.C. Park, J. Mori, J. Geophys. Res. 112, B03302 (2007)

86. E.N. Parker, Astrophys. J. 134, 20 (1961)

87. S. Patsourakos, M.K. Georgoulis, A. Vourlidas, et al., Astrophys. J. 817, 1 (2016)

88. W. Dean Pesnell, B.J. Thompson, P.C. Chamberlin, Solar Phys. 275, 3 (2012)

89. J.L. Phillips, S.J. Bame, W.C. Feldman et al., Science 19, 1030 (1995)

90. V.J. Pizzo, J. Geophys. Res. 83, 5563 (1978)

91. J. Pap, P. Fox (Eds.), Solar variability and its Effects on Climate (Geophysical Monograph, 143, 2004)

92. S.M. Potirakis, T. Asano, M. Hayakawa Entropy 20, 3 (2018)

93. P. Prikryl, D.B. Muldrew, G.J. Sofko, High-speed solar wind auroral electrojets and atmospheric gravity waves: a link to the Earth's atmosphere, in Proceedings International Solar Cycle Studies Symposium "Solar Variability as an Input to the Earth Environment"' (Tatranska Lomnica Slovakia ESA SP, 2003), pp. 23-28

94. P. Prikryl, R. Ghoddousi-Fard, E.G. Thomas et al., Ann. Geophys. 33, 637 (2015)

95. S. Pulinets, K. Boyarchuk, Ionospheric Precursors of Earthquakes (Springer Science \& Business Media, 2004)

96. N.M. Razali, Y.B. Wah, J. Stat. Modeling \& Analytics 2, 21 (2011)

97. R. Rekapalli, Nat. Hazards Earth Syst. Sci. 2, 2851 (2014)

98. C.T. Russell, M. Ginskey, S. Petrinec, G. Le, Geophys. Res. Lett. 19, 1227 (1992)

99. Y. Ruzhin, C. Nomicos, Nat. Hazards 40, 573 (2007)

100. I.G. Richardson, Liv. Rev. Sol. Phys. 1, 15 (2018)

101. I.G. Richardson, H.V. Cane, Space Weather 9, S07005 (2011)

102. I.G. Richardson, H.V. Cane, J. Space Weather Space Clim. 2, A02 (2012)

103. I.G. Richardson, H.V. Cane, J. Space Weather Space Clim. 2, A01 (2012)

104. A.G. Rigas, J. Time Ser. Anal. 13, 441 (1992)

105. E. Robbrecht, S. Patsourakos, A. Vourlidas, ApJ 701, 283 (2009)

106. D.V. Sarafopoulos, J. Atmos. Solar. Terr. Phys. 67, 1427 (2005)

107. I.P. Shestopalov, E.P. Kharin, Russ. J. Earth Sci. 14, ES1002 (2014)

108. N.F. Sidiropoulos, G.C. Anagnostopoulos, V. Rigas, Nat. Hazards Earth Syst. Sci. 11, $1901(2011)$

109. E.C. Stone, L.F. Burlaga, A.C. Cummings et al., The advanced composition explorer Particle Astrophysics - The NASA Cosmic Ray Program for the 1990s and Beyond (1990), https://ntrs.nasa.gov/search.jsp?R=19920037908

110. J.F. Simpson, Earth Planet. Sci. Lett. 3, 417 (1967)

111. E.J. Smith, J.H. Wolfe, Geophys. Res. Lett. 3, 137 (1976)

112. G.A. Sobolev, N.A. Zakrzhevskaya, E.P. Kharin, Phys. Solid Earth Russ. Acad. Sci. 11, $62(2001)$

113. G.A. Sobolev, I.P. Shestopalov, E.P. Kharin, Izvestiya Phys. Solid Earth 34, 603 (1998)

114. A.D. Sytinskiy, Geomagn. Aeron. 3, 120 (1963)

115. A.D. Sytinskii, Izvestiya Phys. Solid Earth 25, 86 (1989)

116. A.D. Sytinskii, Geomagn. Aeron 37, 138 (1997)

117. V. Straser, G. Cataldi, New Conc. Global Tect. J. 3, 141 (2015)

118. M. Tavares, A. Azevedo, Nat. Sci. 3, 436 (2013)

119. M. Tokumaru, M. Kojima, K. Fujiki, J. Geophys. Res. 115, A04102 (2010)

120. V. Tramutoli, R. Corrado, C. Filizzola et al., B. Geofis. Teor. Appl. 56, 167 (2015)

121. K. Tsinganos, Solar and Astrophysical Magnetohydrodynamic Flows (Kluwer, 1995)

122. B.T. Tsurutani, E. Echer, W.D. Gonzalez, J. Atmos. Sol. Terr. Phys. 73, 164 (2011)

123. B.T. Tsurutani, R.L. McPherron, W.D. Gonzalez et al., Geophys. Res. Ser. 167, 1 (2006)

124. B.T. Tsurutani, B.E. Goldstein, E.J. Smith et al., Planet Space Sci. 38, 109 (1990)

125. B.T. Tsurutani, W.D. Gonzalez, Planet Space Sci. 35, 405 (1987)

126. R. Turner, Space Weather 9, S04004 (2011)

127. K. U-Yen, NCGT J. 3, 405 (2015)

128. N. Urata, G. Duma, F. Freund, Open J. Earthq. Res. 7, 39 (2018) 
129. P.I.Y. Velinov, Bulg. Geofiz. Spis. 1, 51 (1975)

130. U. Villante, P. Tiberi, J. Geophys. Res. Space Phys. 121, 4300 (2016)

131. S. Watari, Sol. Phys. 293, 23 (2018)

132. D.F. Webb, R.A. Howard, J. Geophys. Res. 99, 4201 (1994)

133. D.J. Williams, J. Geophys. Res. 71, 1815 (1966)

134. B.A. Yakovlev, Astron. Sbornik Nos 3-4, 152 (1960)

135. S. Yashiro, N. Gopalswamy, G. Michalek et al., J. Geophys. Res. 109, A07105 (2004)

136. H.-Y. Yen, C.-H. Chen, Y.-H. Yeh et al., Earth Planets Space 56, 39 (2004)

137. G. Xystouris, E. Sigala, H. Mavromichalaki, Sol. Phys. 289, 995 (2014)

138. N.A. Zakrzhevskaya, G.A. Sobolev, Phys. Solid Earth Russ. Acad. Sci. 4, 3 (2002)

139. X. Zhang, X. Shen, M. Parrot, Nat. Hazards Earth Syst. Sci. 12, 75 (2012) 